Atmos. Chem. Phys., 13, 1411-1424, 2013

www.atmos-chem-phys.net/13/1411/2013/

doi:10.5194/acp-13-1411-2013

(C) Author(s) 2013. CC Attribution 3.0 License.

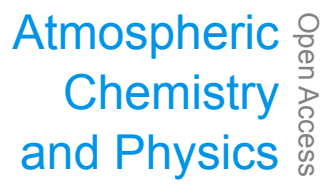

\title{
Daily and hourly chemical impact of springtime transboundary aerosols on Japanese air quality
}

\author{
T. Moreno ${ }^{1}$, T. Kojima ${ }^{2}$, F. Amato ${ }^{3}$, F. Lucarelli ${ }^{4}$, J. de la Rosa ${ }^{5}$, G. Calzolai ${ }^{4}$, S. Nava ${ }^{4}$, M. Chiari ${ }^{4}$, A. Alastuey ${ }^{1}$, \\ X. Querol ${ }^{1}$, and W. Gibbons ${ }^{6}$ \\ ${ }^{1}$ Inst. of Environmental Assessment \& Water Research (IDÆA-CSIC), Jordi Girona 18, 08034 Barcelona, Spain \\ ${ }^{2}$ Dept. of Earth \& Environmental Sciences, Kumamoto University, Kurokami, Kumamoto 860-8555, Japan \\ ${ }^{3}$ TNO Climate, Air and Sustainability, Princetonlaan 6, P.O. Box 80015, 3508 TA Utrecht, The Netherlands \\ ${ }^{4}$ Dept. of Physics and Astronomy, University of Florence, and INFN, Sesto Fiorentino, Florence 50019, Italy \\ ${ }^{5}$ Center for Research in Sustainable Chemistry (CIQSO), University of Huelva, Campus de El Carmen, s/n, 21071 Huelva, \\ Spain \\ ${ }^{6}$ AP 23075, Barcelona 08080, Spain
}

Correspondence to: T. Moreno (teresa.moreno@idaea.csic.es)

Received: 3 July 2012 - Published in Atmos. Chem. Phys. Discuss.: 28 September 2012

Revised: 28 January 2013 - Accepted: 28 January 2013 - Published: 4 February 2013

\begin{abstract}
The regular eastward drift of transboundary aerosol intrusions from the Asian mainland into the NW Pacific region has a pervasive impact on air quality in Japan, especially during springtime. Analysis of 24-h filter samples with Inductively Coupled Plasma Atomic Emission Spectroscopy (ICP-AES) and Mass Spectrometry (ICP-MS), and hourly Streaker with Particle Induced X-ray Emission (PIXE) samples collected continuously for six weeks reveal the chemistry of successive waves of natural mineral desert dust ("Kosa") and metalliferous sulphatic pollutants arriving in western Japan during spring 2011. The main aerosol sources recognised by Positive Matrix Factorization (PMF) analysis of Streaker data are mineral dust and fresh sea salt (both mostly in the coarser fraction $\mathrm{PM}_{2.5-10}$ ), As-bearing sulphatic aerosol $\left(\mathrm{PM}_{0.1-2.5}\right)$, metalliferous sodic particulate matter (PM) interpreted as aged, industrially contaminated marine aerosol, and $\mathrm{ZnCu}$-bearing aerosols. Whereas mineral dust arrivals are typically highly transient, peaking over a few hours, sulphatic intrusions build up and decline more slowly, and are accompanied by notable rises in ambient concentrations of metallic trace elements such as $\mathrm{Pb}, \mathrm{As}, \mathrm{Zn}$, $\mathrm{Sn}$ and $\mathrm{Cd}$. The magnitude of the loss in regional air quality due to the spread and persistence of pollution from mainland Asia is especially clear when cleansing oceanic air advects westward across Japan, removing the continental influence and reducing concentrations of the undesirable metalliferous
\end{abstract}

pollutants by over $90 \%$. Our new chemical database, especially the Streaker data, demonstrates the rapidly changing complexity of ambient air inhaled during these transboundary events, and implicates Chinese coal combustion as the main source of the anthropogenic aerosol component.

\section{Introduction}

The spectacular growth of the Chinese economy in recent years has been accompanied by an equally impressive deterioration in regional air quality (Liu and Mauzerall, 2007; Ohara et al., 2007; Chan and Yao, 2008; Aikawa et al., 2010). The problem is on such a scale that a plume of particulate matter (PM) rich in secondary inorganic compounds (SIC) regularly contaminates millions of square kilometres across the NW Pacific region and beyond (Prospero et al., 2003; Liu et al., 2008; Fairlie et al., 2010; Moreno et al., 2012). The Japanese Archipelago and the Korean Peninsula are especially affected by these transboundary aerosol intrusions. A common scenario is for stagnant anticyclonic conditions over central China concentrating pollutants which later become transported oceanward, sometimes mixing with Gobi Desert dust blown in from the NW (Guo et al., 2004; Ma et al., 2004; Uno et al., 2004; Wang et al., 2004; Chung and Kim, 2008; Zhang et al., 2010; Takahashi et al., 2010). 
The exportation of the resulting aerosol cocktail (Fig. 1) is so frequent as to create in Japan what has been described as a "quasi-permanent" state of regional atmospheric pollution (Lasserre et al., 2008), and has led inevitably to concerns over possible health effects on the Japanese population (e.g. Ichinose et al., 2005; Ueda et al., 2010; Watanabe et al., 2011; Onishi et al., 2012).

In recent years a series of research articles has provided detailed information on these mixtures of natural and anthropogenic PM over East Asia, especially as a result of the 2001 Asian Pacific Regional Aerosol Characterization Experiment (ACE-Asia) (e.g. Uno et al., 2003; Seinfeld et al., 2004; Zhang et al., 2004; Kim et al., 2005; Park et al., 2005 and references therein). Important contributions to the understanding of atmospheric PM chemistry in East Asia also include those using ACE-Asia data (e.g. Arimoto et al., 2004; Satake et al., 2004) as well as detailed observations on individual airborne particles (e.g. Zhang et al., 2003, 2006; Kojima et al., 2006; Tobo et al., 2009) and on transboundary SIC (e.g. Nishikawa et al., 1991; Shimohara et al., 2001; Mori et al., 2003; Trochkine et al., 2003; Zhang et al., 2004; Uno et al., 2007; Aikawa et al., 2010; Fairlie et al., 2010; Kong et al., 2010). There are also papers specifically dealing with the presence of anthropogenic metal-rich particles within Asian atmospheric aerosols (e.g. Kim et al., 2003; Arimoto et al., 2004; Park and Dam, 2010). However there is still a relative paucity of detailed information published on the variations in chemical concentrations of the aerosols people are inhaling during these transboundary pollution events. Many chemical data provide only partial analyses of PM size fractions and/or analyse Total Suspended PM (e.g., Okuda et al., 2008; Yuan et al., 2008; Kim et al., 2009; Kong et al., 2010) rather than strictly the inhalable fraction (Cheng et al., 2005; Chan et al., 2008).

With the above observation in mind in 2010 we conducted a pilot campaign to analyse $24-\mathrm{h} \mathrm{PM}_{10}$ filters collected during three transient transboundary pollution episodes crossing western Japan. The monitoring site used was in Kumamoto on the island of Kyushu in SW Japan, in the area most frequently visited by transboundary PM outflows from the Asian mainland. Our results confirmed the pronounced bimodality and inhomogeneity between natural and anthropogenic PM in East Asian transboundary aerosol intrusions (Moreno et al., 2012). Furthermore, although the number of filters analysed in the pilot study was small, it was nevertheless enough to demonstrate the highly metalliferous and chemically complex nature of sulphatic plumes arriving from industrial China. Such plumes cross Japan, creating regional pollution clouds that dissipate only slowly due to the dominance of atmospherically persistent submicron accumulation mode PM. It was this aspect of the chemical data, rather than the already well-characterised nature of the "natural" GobiDesert derived Kosa intrusions, which seemed to us in more urgent need of further study.

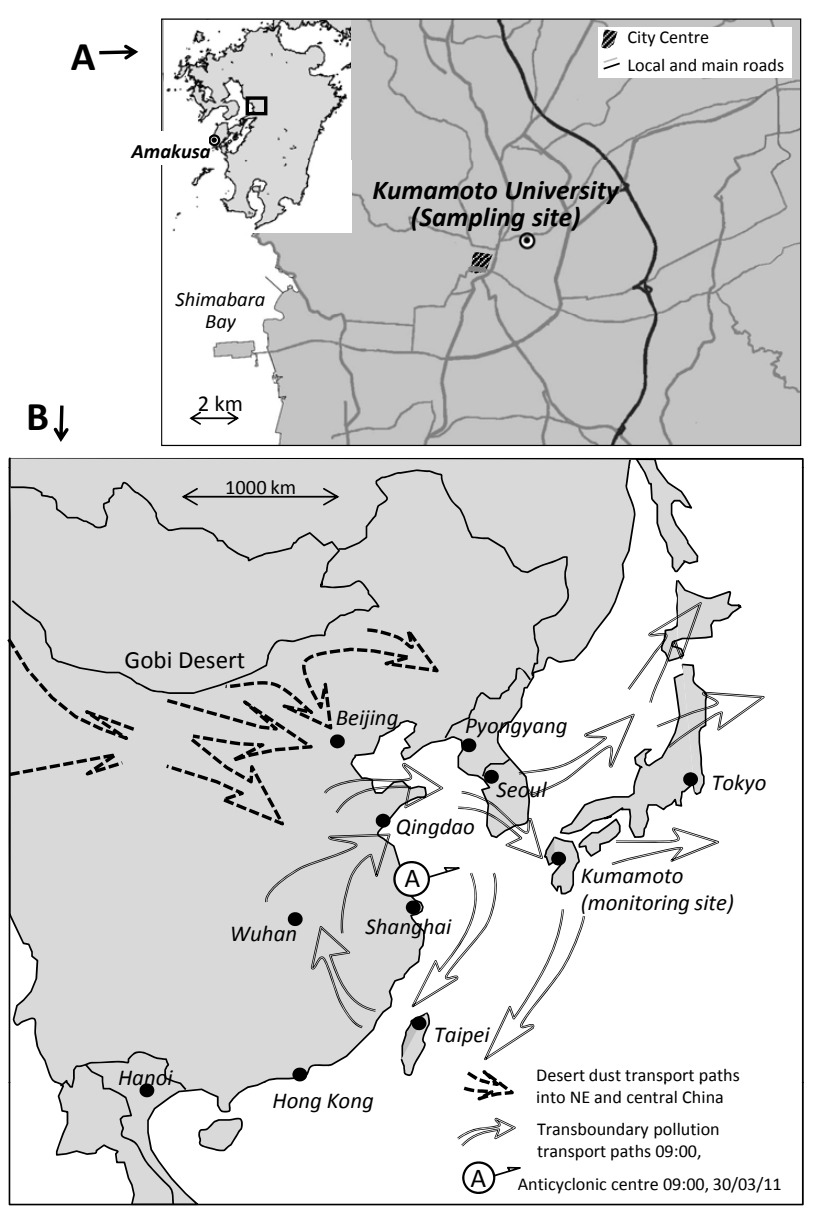

Fig. 1. Top: a simplified map of the area around the sampling site (rectangle in the inset) showing major roads including the Kyushu Expressway (darker line). Bottom: location map showing Kumamoto monitoring site and overviewing the area affected by the transboundary expulsion of anthropogenic aerosols from China into the NW Pacific region. Arrows depict typical atmospheric circulation patterns driving the pollution eastward out from the mainland, in this case by an anticyclone centred near Shanghai during the sampling period at the end of March 2011. The main source areas of springtime desert dust intrusions into the area are also shown (Wang et al., 2004).

In this context this manuscript moves forward by presenting a new database collected during a 6-week period of continuous hourly and daily measurements in Kumamoto, SW Japan. The study adopts an unusually multi-analytical approach by integrating results from Particle Induced X-ray Emission (PIXE), Inductively Coupled Plasma Mass Spectrometry/Atomic Emission (ICPMS/AE) spectroscopy, chromatography and thermal-optical transmission methods, allowing comparison between hourly (Streaker) and 24-h (filter) data. Such data are unprecedented in the chemical detail they offer on Japanese air chemistry during transboundary aerosol inflows. The Streaker data in particular provides over 
1000 hourly measurements of major and trace elements for each of the coarse and fine size fractions, a very large number which allows us to perform a positive matrix factorization (PMF) analysis and so separate differently sourced components within the aerosol mixture.

The primary aim of this new campaign was thus to provide further insight into the complex atmospheric mixing processes and changes in air quality that occur within transboundary receptor areas lying immediately downstream from East Asian aerosol outflows. Using a time resolution measuring hourly as well as daily fluctuations allowed us to compare polluted and "clean" days, and to develop a clearer idea of the reality of what people are inhaling during these pollution episodes. This database would allow us to resolve in more detail than any previous study the speed, duration and chemistry of different types of pollution episodes reaching Japan. We consider these kind of previously unavailable data as prerequisite information for those tasked with provided health advice to affected populations prior to the predicted arrival of transboundary atmospheric pollutants.

\section{Methodology}

Samples were obtained in March and April 2011 at the top of a nine-storey building within the Kumamoto University precinct on the island of Kyushu in Western Japan (Fig. 1) approximately midway between Tokyo (ca. $1000 \mathrm{~km}$ ENE) and Shanghai (ca. $1000 \mathrm{~km} \mathrm{WSW).} \mathrm{Kumamoto} \mathrm{city} \mathrm{is} \mathrm{not} \mathrm{im-}$ pacted by any nearby heavy industrial point sources, making it an excellent location to observe the arrival of transboundary aerosol intrusions. The monitoring site can be classified as an urban background site influenced to a limited extent by road traffic emissions from a city centre arterial road $1400 \mathrm{~m}$ to the west and a minor two-lane road crossing the University area. We had previously "ground truth tested" our Kumamoto monitoring site by comparing $\mathrm{SO}_{4}$ concentrations obtained by ion chromatography (IC) of Kumamoto University filter samples with that by Sulfate Particle Analyzer (SPA) at Amakusa, a remote coastal on the western coast of Japan, $90 \mathrm{~km}$ SW of Kumamoto (Nagatani et al., 2012). The data for 2009 and 2010 correlate well $\left(R^{2}=0.89\right)$ and demonstrated that sulfate concentration in Kumamoto City was not significantly higher than those in Amakusa.

Air quality data from 33 air quality monitoring stations (urban/suburban and rural areas) operated by the Kumamoto Prefectural Government were compared with those measured by us at Kumamoto University and demonstrated a very good correlation during the whole campaign (http://taiki.pref. kumamoto.jp/kumamoto-taiki/index.htm). This same pattern was also observed with data continuously measured at Amakusa and emphasised the dominant influence of transboundary aerosol across the whole area, with only a relatively minor contribution to PM levels from local Kumamoto sources away from busy roads. A similar result is obtained from unpublished data on Optical Particle Counter data measured at Amakusa during the same period, which shows timeseries patterns strikingly similar to those observed by us in Kumamoto during our campaign (D. Zhang, Kumamoto Prefectural University, personal communication, 2012). As further background to our study, a recent paper by Kaneyasu et al. (2011) compares $\mathrm{PM}_{2.5}$ concentrations during 2009 and 2010 in Fukuoka (the largest city in Kyushu, $90 \mathrm{~km} \mathrm{~N}$ of Kumamoto), Nagasaki (medium-sized city $75 \mathrm{~km}$ west of Kumamoto), and Fukue Island (remote site $100 \mathrm{~km}$ west of Nagasaki). It demonstrates that from March to May 2011, $\mathrm{PM}_{2.5}$ concentrations in Fukue Island were higher than in Fukuoka (Kaneyasu et al., 2011). So it is clear that air quality in Kyushu is affected more by transboundary aerosols than by local emissions, even in Fukuoka, a city far more highly populated and industrialized than Kumamoto. In the light of all these observations we are confident that our rooftop $\mathrm{Ku}-$ mamoto monitoring site is well suited to measuring transboundary inflows into western Japan.

We measured hourly element concentrations continuously from 17 March to 28 April using a Streaker sampler collecting hourly aerosol samples in two size ranges $(0.1-2.5 \mu \mathrm{m}$ and $2.5-10 \mu \mathrm{m})$ at an air flow rate of $1 \mathrm{~L} \mathrm{~min}^{-1}$ that were then analyzed by Particle Induced X-Ray Emission (PIXE, see Lucarelli et al., 2011 for details) at the LABEC-INFN facility in Florence (based on a 3 MV Tandetron accelerator, where an external beam set-up is fully dedicated to atmospheric aerosol studies). For daily samples we used a SIBATA HV-1000F high volume PM sampler $\left(60 \mathrm{~m}^{3} \mathrm{~h}^{-1}\right)$ which excluded particles larger than $10 \mu \mathrm{m}$. Twenty-four hour filter samples were collected from 22 March until 28 April (from 12:00 p.m. local time). Quartz fibre filters (ADVANTEC QR-100) were conditioned (30-40\% relative humidity over $48 \mathrm{~h}$ ) and weighed before and after sampling to determine $24 \mathrm{~h} \mathrm{PM}_{10}$ concentrations by standard gravimetric procedures. Once the gravimetric determination was performed the filters were treated and analysed for the determination of the chemical composition of $\mathrm{PM}_{10}$. One quarter of each filter was acid digested (HF: $\mathrm{HNO}_{3}: \mathrm{HClO}_{4}$, with a mixture of 2.5:1.25:1.25 mL, kept at $90^{\circ} \mathrm{C}$ in a Teflon reactor during $6 \mathrm{~h}$, driven to dryness and re-dissolved with $1.25 \mathrm{~mL}$ $\mathrm{HNO}_{3}$ to make up a volume of $25 \mathrm{~mL}$ with water) for the chemical analysis using ICP-AES and ICP-MS. To assure the quality of the analytical procedure $5 \mathrm{mg}$ of the NIST-1633b (fly ash) reference material loaded on a $1 / 4$ quartz microfibre filter were also analysed. Detection limit and accuracy of the techniques were estimated as $0.18 \mathrm{ng} \mathrm{m}^{-3}$ and $1-3 \%$ respectively for ICP-AES, and $0.007 \mathrm{ng} \mathrm{m}^{-3}$ and $0-7 \%$ respectively for ICP-MS. The detection limits for $\mathrm{Zr}$ and $\mathrm{Hf}$ are higher $\left(0.05 \mathrm{ng} \mathrm{m}^{-3}\right)$. Another $1 / 4$ of each filter was water leached $\left(6 \mathrm{~h}\right.$ at $60^{\circ} \mathrm{C}$, preceded by incubation in an ultrasound bath for $10 \mathrm{~min}$, in $50 \mathrm{~mL}$ sealed PVC bottles) for the determination of soluble ion concentrations by ion chromatography (sulphate, nitrate and chloride) and ion selective electrode (ammonium), allowing an average detection 
limit for the analysed components of $25-30 \mathrm{ng} \mathrm{m}^{-3}$. A portion $\left(1.5 \mathrm{~cm}^{2}\right)$ of the remaining half of each filter was also used for the determination of organic and elemental carbon (OC and EC, respectively) by a thermal-optical transmission technique (Birch and Cary, 1996) using a Sunset Laboratory OC-EC Analyser with the EUSAAR-2 standard temperature programme. The accuracy of the equipment is in the rage of $5-10 \%$, depending on the relative quantities of OC and EC on the filter, and the detection limit $0.2 \mu \mathrm{g} \mathrm{m}^{-3}$ for both OC and EC. The sum OC $+\mathrm{EC}$ is $C_{\text {total }}$. The $\mathrm{OM}+\mathrm{EC}$ (organic matter plus elemental carbon) value was obtained after applying a 1.6 factor to the OC concentrations (Turpin et al., 2000).

$\mathrm{SiO}_{2}$ and $\mathrm{CO}_{3}^{2-}$ were indirectly determined on the basis of empirical factors $\left(\mathrm{Al} * 1.89=\mathrm{Al}_{2} \mathrm{O}_{3}, 3 * \mathrm{Al}_{2} \mathrm{O}_{3}=\mathrm{SiO}_{2}\right.$ and $1.5^{*} \mathrm{Ca}+2.5^{*} \mathrm{Mg}=\mathrm{CO}_{3}^{2-}$, see Querol et al., 2001). Blank field filters were used for every stock purchased for sampling and analysed in the same batches of their respective filter samples. The corresponding blank concentrations were subtracted from each sample.

A Positive Matrix Factorization (PMF, Paatero and Tapper, 1994) was performed on the two data matrices of concentrations and uncertainties of hourly Streaker samples values. This provides an estimation of the main sources contributing to the measured PM by weighting each data point by its analytical uncertainty and solving the following equation:

$\mathbf{x}_{i j}=\sum_{h=1}^{p} \mathbf{g}_{i h} \mathbf{f}_{h j}+\mathbf{e}_{i j}$

The model uses the least squares method where the indices $i, j$ and $h$ refer to the number of samples, chemical components and factors respectively, while the matrices $\mathbf{x}, \mathbf{g}$ and $\mathbf{f}$ refer to the concentration data, factor contribution (or factor scores) and factor profiles (or factor loading), respectively. The matrix $\mathbf{e}$ is the matrix of residuals defined as:

$\mathbf{e}_{i j}=\mathbf{x}_{i j}-\sum_{h=1}^{p} \mathbf{g}_{i h} \mathbf{f}_{h j}$

The matrices $\mathbf{g}$ and $\mathbf{f}$ are found by minimizing the loss function $Q$ defined as the sum of the squared residuals weighted by the uncertainty $u_{i j}$ associated with the each data point:

$$
Q=\sum_{i} \sum_{j}\left[\frac{\mathbf{e}_{i j}}{u_{i j}}\right]^{2}
$$

Individual estimates of the concentration errors were calculated following the methodology described by Amato et al. (2009). The uncertainty estimate provides a basis to separate species which retain a significant signal from the ones dominated by noise. This principle is based on the signalto-noise $S / N$ ratio described by Paatero and Hopke (2003). However, due to the sensibility of $S / N$ to sporadic values much higher than the level of noise, the percentage of data above detection limit (ADL) was used as a complementary criterion. Given the relatively low number of samples, species were selected to perform the source apportionment study. The selection was based on the Signal to Noise $(S / N)$ ratio and \% of data above detection limit criteria (Amato et al., 2009).

The transport pathways of air-masses into the Kumamoto area during the monitoring period were assessed using the HYSPLIT-model (Draxler and Rolph, 2003), with vertically modelled transport back-trajectories being calculated for 5 days at 750, 1500 and $2500 \mathrm{~m}$ a.s.l. In addition dust and sulphate maps forecasted by the Chemical Weather Forecasting System (CFORS) were obtained from the website of the National Institute for Environmental Studies (http: //www-cfors.nies.go.jp/ cfors/) from 2 March onwards (before this date the system had been disrupted by the Tōhoku Earthquake on 11 March). CFORS calculates distributions of Asian dust and anthropogenic sulphate aerosols every three hours, the results being uploaded on the website every day (Uno et al., 2003; Satake et al., 2004), and is widely referred to as a source of real-time information on movements of dust and pollution plumes over Asia. Finally, weather conditions (wind velocity and direction, precipitation, relative humidity and ambient temperature) were obtained from the Kumamoto Meteorological Observatory, located about $2 \mathrm{~km}$ west of the sampling site.

\section{Results}

Our PMF analysis of $>1000$ analyses obtained from the Streaker coarse fraction $\left(\mathrm{PM}_{2.5-10}\right)$ data allows us to detect 4 main source factors (Fig. 2a) these being: (1) Mineral dust with major elements $\mathrm{Si}, \mathrm{Al}, \mathrm{Ca}, \mathrm{Fe}, \mathrm{K}, \mathrm{Mg}$ and a range of trace elements that includes several metals such as Ti, Mn, $\mathrm{Cu}$; (2) Metalliferous sodic aerosol, accounting for $55 \%$ of $\mathrm{Na}$ and significant proportions of $\mathrm{S}, \mathrm{Mg}, \mathrm{Ca}, \mathrm{Cu}, \mathrm{Zn}$ and Sr; (3) Fresh marine aerosol, explaining almost $90 \%$ of $\mathrm{Cl}$ concentrations and including also $\mathrm{Na}$ and smaller amounts of $\mathrm{Mg}$; (4) $\mathrm{ZnCu}$ aerosol tentatively attributed to road traffic emissions and associated with mineral elements $(\mathrm{Si}, \mathrm{Al}$, $\mathrm{Mg}, \mathrm{Ca}, \mathrm{K}, \mathrm{Ti})$ related to dust resuspension processes, making both profiles (mineral dust and $\mathrm{ZnCu}$ aerosol) very similar.

With regard to the fine aerosol fraction $\left(\mathrm{PM}_{0.1-2.5}\right)$ once again the best PMF solution is obtained with 4 factors (Fig. 2b), although with distinct differences from those of the coarser fraction: (1) As-bearing sulphatic aerosol ("China plume"), dominated by $\mathrm{S}$ and As but also with smaller amounts of many other elements (K, Se, Sr, Ti, Mn, Fe, Zn); (2) Metalliferous sodic aerosol, which in this size fraction accounts for $>80 \%$ of $\mathrm{Na}$, associated with $\mathrm{Se}, \mathrm{Sr}, \mathrm{Mg}, \mathrm{Cu}$, $\mathrm{Ca}$ and $\mathrm{S}$ (with no $\mathrm{Cl}$ being detected due to low concentrations in this size fraction); (3) Mineral dust, including $\mathrm{Al}$ ( $\mathrm{Si}$ not analysed), $\mathrm{Ca}, \mathrm{Fe}, \mathrm{K}$, and $\mathrm{Mg}$; (4) $\mathrm{Zn}$-Cu aerosol again 

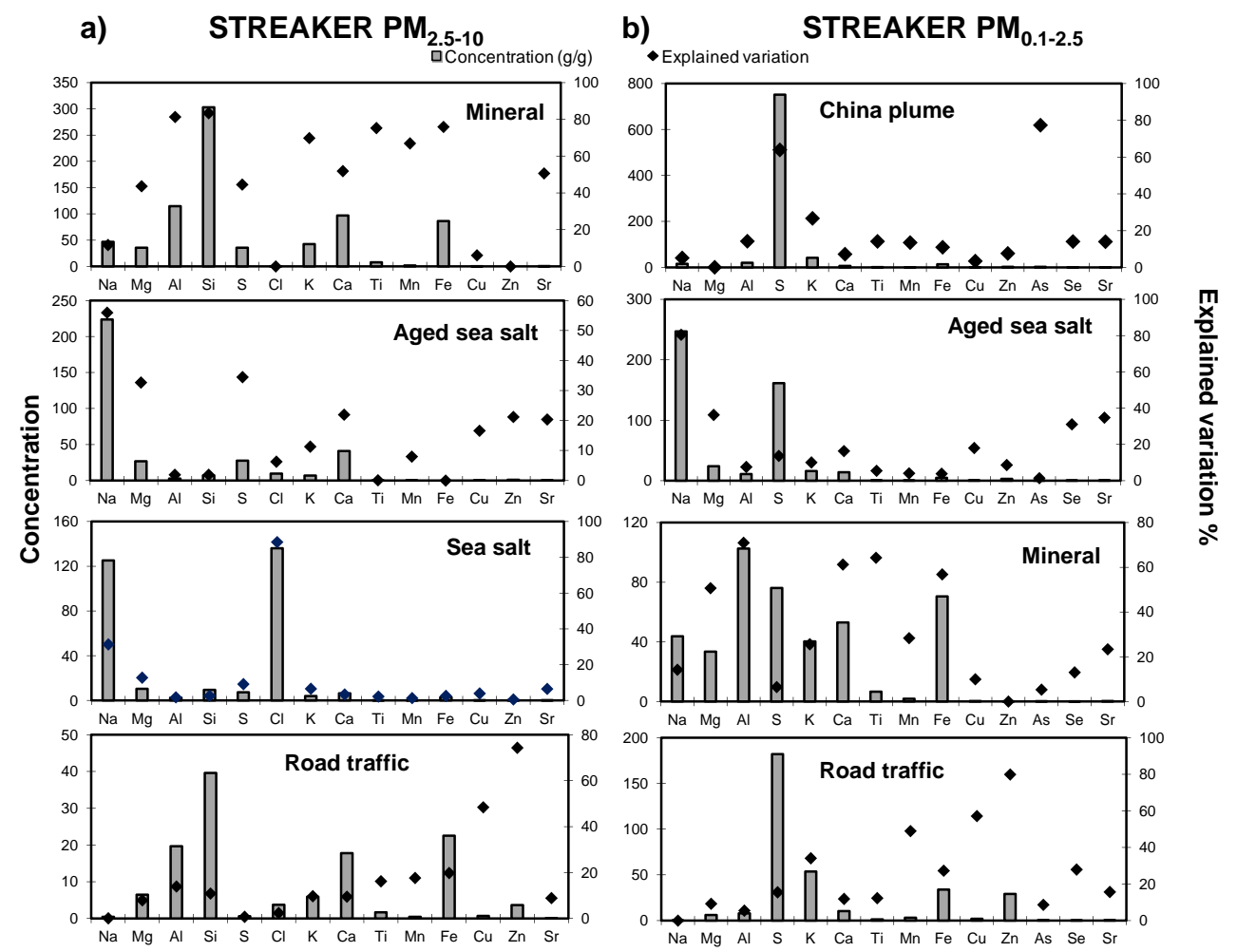

Fig. 2. Chemical profile for each factor identified by Positive Matrix Factorization (PMF) for hourly Streaker samples showing concentration histograms and the explained variation for each element.

attributed at least in part to road traffic, explaining most of $\mathrm{Zn}$ and $\mathrm{Cu}$ (as seen in the coarse fraction), but also associated with $\mathrm{Mn}, \mathrm{K}, \mathrm{Se}, \mathrm{Fe}, \mathrm{S}$ and $\mathrm{Sr}$ suggesting a more mixed source for this very fine metalliferous component.

The chemical results for the $37 \mathrm{PM}_{10}$ filter samples reveal considerable daily variation in PM concentrations. The full analyses are provided in Supplementary Information Appendix on Table $\mathrm{S} 1$ but a selected sample group representing the main chemical variation is provided in Table 1. The ICPAES and MS database confirms predictions made by CFORS and HYSPLIT data and identifies two extended periods when transboundary sulphatic air pollution was most prominent (28 March-3 April and 9-18 April, Fig. 3), separated by a cleansing episode induced by the advection of oceanic air across Japan. Levels of nss- $\mathrm{SO}_{4}^{2-}$ rose to peaks exceeding $15 \mu \mathrm{g} \mathrm{m}^{-3}$ during the two pollution events (samples 290311, 150411 and 170411 on Table 1), but fell to a minimum of $<2 \mu \mathrm{g} \mathrm{m}^{-3}$ during the intervening clean period (040411 and 190411 Table 1). The data also indicate the presence of elevated levels of mineral dust in several samples (e.g. 220311, 280311, 090411, 100411, 210411 Table 1, Fig. 3), recorded by increased concentrations of typically "geological" major elements such as $\mathrm{Al}, \mathrm{Ca}, \mathrm{Fe}$. Levels of $\mathrm{Ti}$, a reliable tracer for mineral dust, rise above $55 \mathrm{ng} \mathrm{m}^{-3}$ during these Kosa events (Table 1).
The Streaker data are summarized in Fig. $4 \mathrm{a}-\mathrm{d}$ and also clearly identify the two main pollution episodes (280311030411 and 09-180411) separated by a phase of oceanic advection. Increased levels of fine sulphate (represented by $\mathrm{S}$ $\mathrm{PM}_{0.1-2.5}$ on Fig. 4a) are typically accompanied by higher concentrations of the more toxic metallic elements (e.g. Pb, As on Fig. 4b). A somewhat contrasting pattern of fluctuations in the natural mineral dust component is represented by concentrations of $\mathrm{Al}$ and $\mathrm{Ca}$ (hourly Streaker data) plotted in Fig. 4c, revealing a series of transient peaks of these crustal elements as waves of Gobi dust crossed Kyushu during the 6-week sampling period. With reference to Fig. 4 we now consider these air quality events in more detail by subdividing the results from the sampling period into five distinct phases.

\subsection{Phase 1: 17-27 March}

The first 10 days of the Streaker campaign were characterized by NW winds feeding in transient, dilute waves of aerosols from the Asian mainland. $\mathrm{PM}_{10}$ levels stayed below $40 \mu \mathrm{g} \mathrm{m}^{-3}$, with both nss-SO $\mathrm{SO}_{4}^{2-}$ and $\mathrm{NO}_{3}^{-}$concentrations being confined to a narrow range $\left(3-6 \mu \mathrm{g} \mathrm{m}^{-3}\right)$, and amounts of mineral dust $\left(5-14 \mu \mathrm{g} \mathrm{m}^{-3}\right)$ varied depending on the timing of the arrival of Gobi intrusions. Whereas the first desert dust event recorded (19 March) 
Table 1. Daily, average, maximum, and standard deviation values for elemental concentrations analysed in selected $\mathrm{PM}_{10}$ gravimetric filters collected in Kumamoto March-April 2011 (see text for details), including average values for the full dataset from 22 March to 28 April. Each sample bears the date when 24-h sampling started at midday and therefore includes the first half of the following day. PM and major element concentrations are in $\mu \mathrm{g} \mathrm{m}^{-3}$, trace elemental concentrations in $\mathrm{ng} \mathrm{m}^{-3}$. Mineral $=\mathrm{CO}_{3}^{2-}+\mathrm{SiO}_{2}+\mathrm{Al}_{2} \mathrm{O}_{3}+\mathrm{Ca}+\mathrm{Fe}+\mathrm{K}+\mathrm{Mg}$; $\mathrm{OM}+\mathrm{EC}=$ Organic matter + elemental carbon; $\mathrm{SIC}=\mathrm{NH}_{4}^{+}+\mathrm{NO}_{3}^{-}+\mathrm{SO}_{4}^{2-} ;$ Marine $=\mathrm{Na}+\mathrm{Cl}$.

\begin{tabular}{|c|c|c|c|c|c|c|c|c|c|c|c|c|c|c|c|c|c|c|}
\hline Date & 220311 & 230311 & 240311 & 280311 & 290311 & 40411 & 80411 & 90411 & 100411 & 150411 & 160411 & 170411 & 190411 & 210411 & 240411 & average & $\max$ & std. dev \\
\hline \multicolumn{19}{|l|}{$\mu \mathrm{g} \mathrm{m}^{-3}$} \\
\hline $\mathrm{PM}_{10}$ & 39.7 & 22.9 & 36.4 & 47.7 & 65.7 & 22.9 & 27.7 & 45.1 & 52.3 & 54.9 & 64.0 & 54.7 & 18.6 & 47.6 & 36.3 & 37.6 & 65.7 & 12.4 \\
\hline $\mathrm{OM}+\mathrm{EC}$ & 7.0 & 6.9 & 11.2 & 11.0 & 11.8 & 4.5 & 4.3 & 8.7 & 10.4 & 9.0 & 18.2 & 13.4 & 5.1 & 11.7 & 9.1 & 9.0 & 18.2 & 3.1 \\
\hline $\mathrm{OC}$ & 4.0 & 4.0 & 6.1 & 6.2 & 6.8 & 2.6 & 2.5 & 5.4 & 6.1 & 5.2 & 10.3 & 7.9 & 2.8 & 6.8 & 5.2 & 5.1 & 10.3 & 1.8 \\
\hline EC & 0.8 & 0.5 & 1.2 & 1.0 & 0.8 & 0.4 & 0.4 & 0.5 & 0.8 & 0.7 & 1.3 & 0.8 & 0.6 & 0.9 & 0.6 & 0.7 & 1.3 & 0.2 \\
\hline $\mathrm{CO}_{3}^{=}$ & 2.2 & 0.9 & 1.7 & 1.8 & 1.0 & 0.9 & 1.1 & 2.4 & 2.1 & 1.3 & 1.4 & 1.3 & 0.6 & 1.9 & 0.5 & 1.2 & 2.4 & 0.5 \\
\hline $\mathrm{SiO}_{2}$ & 6.8 & 2.4 & 5.0 & 5.5 & 3.7 & 4.7 & 4.3 & 8.3 & 7.2 & 3.6 & 4.4 & 4.2 & 1.4 & 8.5 & 1.4 & 4.1 & 8.5 & 1.6 \\
\hline $\mathrm{Al}_{2} \mathrm{O}_{3}$ & 2.3 & 0.8 & 1.7 & 1.8 & 1.2 & 1.6 & 1.4 & 2.8 & 2.4 & 1.2 & 1.5 & 1.4 & 0.5 & 2.8 & 0.5 & 1.4 & 2.8 & 0.6 \\
\hline $\mathrm{Ca}$ & 0.8 & 0.3 & 0.7 & 0.8 & 0.4 & 0.4 & 0.4 & 0.9 & 0.8 & 0.4 & 0.5 & 0.5 & 0.2 & 0.8 & 0.2 & 0.5 & 0.9 & 0.2 \\
\hline $\mathrm{Fe}$ & 0.8 & 0.3 & 0.7 & 0.8 & 0.6 & 0.6 & 0.5 & 1.0 & 0.9 & 0.5 & 0.6 & 0.6 & 0.2 & 0.9 & 0.2 & 0.5 & 1.0 & 0.2 \\
\hline $\mathrm{K}$ & 0.5 & 0.2 & 0.4 & 0.5 & 0.5 & 0.2 & 0.3 & 0.7 & 0.7 & 0.6 & 0.7 & 0.6 & 0.1 & 0.6 & 0.4 & 0.4 & 0.7 & 0.2 \\
\hline $\mathrm{Na}$ & 0.6 & 0.4 & 0.3 & 0.4 & 0.3 & 0.4 & 0.3 & 0.5 & 0.5 & 0.9 & 1.1 & 1.0 & 0.6 & 0.6 & 0.3 & 0.6 & 1.1 & 0.3 \\
\hline $\mathrm{Mg}$ & 0.4 & 0.2 & 0.3 & 0.3 & 0.1 & 0.1 & 0.2 & 0.5 & 0.4 & 0.3 & 0.3 & 0.3 & 0.1 & 0.3 & 0.1 & 0.2 & 0.5 & 0.1 \\
\hline $\mathrm{Cl}$ & 0.8 & 0.2 & 0.5 & 0.4 & 0.8 & 1.2 & n.a. & 0.2 & 0.1 & 0.4 & 0.7 & 0.6 & 0.5 & 0.2 & 0.4 & 0.5 & 1.2 & 0.3 \\
\hline $\mathrm{NO}_{3}^{-}$ & 4.4 & 3.9 & 6.0 & 8.1 & 16.1 & 1.8 & 1.3 & 4.2 & 4.6 & 6.2 & 4.4 & 3.7 & 2.0 & 2.9 & 5.0 & 4.6 & 16.1 & 2.9 \\
\hline $\mathrm{SO}_{4}^{\underline{\underline{3}}}$ & 5.1 & 3.2 & 3.7 & 9.2 & 16.6 & 1.9 & 4.1 & 4.6 & 7.0 & 18.0 & 11.2 & 17.0 & 2.0 & 7.3 & 10.5 & 7.0 & 18.0 & 4.1 \\
\hline nss-SO $=$ & 5.0 & 3.1 & 3.7 & 9.2 & 16.5 & 1.9 & 4.0 & 4.5 & 7.0 & 17.9 & 11.1 & 16.9 & 1.9 & 7.2 & 10.4 & 6.9 & 17.9 & 4.1 \\
\hline $\mathrm{NH}_{4}^{+}{ }^{4}$ & 0.6 & 0.5 & 0.7 & 1.5 & 4.3 & 0.3 & 0.9 & 0.8 & 1.1 & 1.7 & 1.8 & 1.7 & 0.2 & 0.7 & 1.5 & 1.0 & 4.3 & 0.8 \\
\hline \multicolumn{19}{|l|}{$\mathrm{ng} \mathrm{m}^{-3}$} \\
\hline $\mathrm{Li}$ & 0.92 & 0.31 & 0.69 & 0.97 & 0.92 & 0.40 & 0.55 & 1.15 & 1.13 & 0.70 & 0.76 & 0.72 & 0.15 & 0.83 & 0.30 & 0.57 & 1.15 & 0.25 \\
\hline $\mathrm{Be}$ & 0.06 & 0.04 & 0.03 & 0.04 & 0.03 & 0.04 & 0.04 & 0.06 & 0.07 & 0.04 & 0.04 & 0.04 & 0.02 & 0.06 & 0.03 & 0.04 & 0.07 & 0.01 \\
\hline $\mathrm{Sc}$ & 0.84 & n.a. & 0.82 & 0.03 & 0.38 & 2.69 & 0.24 & 2.61 & 4.12 & 0.10 & 1.00 & 0.73 & 0.42 & 1.02 & 1.79 & 1.10 & 4.12 & 0.86 \\
\hline $\mathrm{Ti}$ & 58.08 & 28.18 & 55.06 & 60.66 & 43.66 & 50.45 & 43.59 & 87.21 & 77.30 & 41.64 & 49.82 & 44.50 & 17.98 & 91.01 & 17.87 & 45.10 & 91.01 & 16.15 \\
\hline $\mathrm{V}$ & 4.72 & 3.16 & 3.44 & 3.71 & 3.52 & 2.55 & 3.44 & 4.37 & 5.14 & 8.57 & 4.65 & 5.19 & 1.34 & 3.65 & 2.83 & 3.59 & 8.57 & 1.34 \\
\hline $\mathrm{Cr}$ & 2.82 & 1.84 & 4.02 & 4.80 & 4.90 & $<$ d.1. & 1.49 & 2.71 & 3.06 & 5.63 & 2.28 & 1.93 & 0.42 & 1.90 & 1.11 & 2.15 & 5.63 & 1.46 \\
\hline $\mathrm{Mn}$ & 29.19 & 14.38 & 30.58 & 35.45 & 32.31 & 19.23 & 20.97 & 30.85 & 36.86 & 27.50 & 25.90 & 23.57 & 8.13 & 29.51 & 12.85 & 21.61 & 36.86 & 7.91 \\
\hline Co & 0.36 & 0.20 & 0.34 & 0.38 & 0.31 & 0.25 & 0.23 & 0.42 & 0.42 & 0.28 & 0.27 & 0.25 & 0.08 & 0.35 & 0.11 & 0.24 & 0.42 & 0.08 \\
\hline $\mathrm{Ni}$ & 2.68 & 2.05 & 4.33 & 4.58 & 4.46 & 3.12 & 1.85 & 2.19 & 2.78 & 4.39 & 2.99 & 3.12 & 1.18 & 1.63 & 1.47 & 2.81 & 4.90 & 1.20 \\
\hline $\mathrm{Cu}$ & 5.68 & 4.60 & 7.64 & 8.93 & 9.69 & 4.24 & 3.42 & 5.68 & 9.84 & 10.69 & 7.29 & 6.46 & 3.26 & 6.71 & 5.82 & 5.96 & 11.12 & 2.28 \\
\hline $\mathrm{Zn}$ & 67.15 & 73.18 & 88.72 & 120.21 & 143.00 & 34.60 & 30.03 & 58.59 & 112.05 & 130.90 & 97.85 & 87.91 & 23.14 & 56.53 & 70.14 & 71.53 & 151.49 & 38.14 \\
\hline $\mathrm{Ga}$ & 0.63 & 0.31 & 0.52 & 0.84 & 1.24 & 0.29 & 0.40 & 0.63 & 0.75 & 0.68 & 0.67 & 0.64 & 0.09 & 0.54 & 0.38 & 0.47 & 1.24 & 0.23 \\
\hline As & 3.41 & 2.02 & 2.76 & 5.89 & 5.33 & 1.75 & 1.10 & 2.62 & 3.21 & 5.31 & 5.06 & 4.48 & 0.52 & 2.18 & 1.95 & 2.61 & 5.89 & 1.52 \\
\hline $\mathrm{Se}$ & 0.77 & 0.36 & 0.80 & 1.53 & 0.96 & 0.53 & 0.69 & 0.98 & 1.61 & 0.59 & 0.76 & 0.53 & 0.35 & 0.85 & 0.18 & 0.76 & 1.69 & 0.37 \\
\hline $\mathrm{Rb}$ & 2.48 & 0.97 & 1.76 & 2.47 & 2.54 & 0.91 & 1.34 & 3.09 & 3.24 & 2.56 & 2.52 & 2.29 & 0.38 & 2.24 & 1.44 & 1.72 & 3.24 & 0.79 \\
\hline $\mathrm{Sr}$ & 6.15 & 2.25 & 4.02 & 4.79 & 3.33 & 2.41 & 3.67 & 7.40 & 6.26 & 3.68 & 3.71 & 3.71 & 1.05 & 5.31 & 1.44 & 3.27 & 7.40 & 1.41 \\
\hline $\mathrm{Cd}$ & 0.66 & 0.31 & 0.39 & 0.77 & 1.11 & 0.26 & 0.33 & 0.44 & 0.65 & 1.13 & 0.89 & 0.88 & 0.15 & 0.31 & 0.40 & 0.47 & 1.13 & 0.28 \\
\hline $\mathrm{Sn}$ & 1.28 & 0.84 & 1.58 & 2.40 & 3.02 & 0.81 & 0.96 & 1.42 & 2.32 & 3.88 & 2.50 & 2.44 & 0.51 & 1.56 & 1.29 & 1.55 & 3.88 & 0.77 \\
\hline $\mathrm{Sb}$ & 1.13 & 0.95 & 1.84 & 2.42 & 2.81 & 0.62 & 0.92 & 1.51 & 2.69 & 3.01 & 2.45 & 2.02 & 0.87 & 1.69 & 1.53 & 1.68 & 6.86 & 1.13 \\
\hline Cs & 0.34 & 0.14 & 0.24 & 0.38 & 0.43 & 0.17 & 0.16 & 0.42 & 0.48 & 0.41 & 0.33 & 0.31 & 0.04 & 0.23 & 0.25 & 0.25 & 0.48 & 0.13 \\
\hline $\mathrm{Ba}$ & 13.95 & 8.97 & 12.89 & 19.39 & 11.15 & 5.04 & 8.83 & 17.62 & 19.88 & 10.29 & 9.92 & 9.09 & 4.20 & 15.31 & 7.79 & 10.22 & 23.48 & 4.41 \\
\hline $\mathrm{La}$ & 0.77 & 0.33 & 0.63 & 0.68 & 0.37 & 0.24 & 0.49 & 0.93 & 0.94 & 0.48 & 0.58 & 0.58 & 0.15 & 0.70 & 0.25 & 0.45 & 0.94 & 0.20 \\
\hline $\mathrm{Ce}$ & 1.47 & 0.62 & 1.11 & 1.31 & 0.69 & 0.63 & 0.96 & 1.87 & 1.88 & 0.90 & 0.97 & 0.94 & 0.40 & 1.39 & 0.55 & 0.87 & 1.88 & 0.38 \\
\hline $\mathrm{Pr}$ & 0.17 & 0.07 & 0.11 & 0.14 & 0.06 & 0.06 & 0.11 & 0.22 & 0.19 & 0.07 & 0.08 & 0.08 & 0.01 & 0.15 & 0.03 & 0.08 & 0.22 & 0.05 \\
\hline $\mathrm{Nd}$ & 0.56 & 0.24 & 0.33 & 0.43 & 0.17 & 0.15 & 0.37 & 0.71 & 0.64 & 0.27 & 0.32 & 0.30 & 0.08 & 0.55 & 0.12 & 0.28 & 0.71 & 0.16 \\
\hline $\mathrm{Sm}$ & 0.13 & 0.06 & 0.03 & 0.05 & 0.01 & $<$ d.1. & 0.08 & 0.15 & 0.13 & 0.06 & 0.07 & 0.06 & 0.02 & 0.13 & 0.03 & 0.06 & 0.15 & 0.04 \\
\hline Eu & 0.04 & 0.03 & 0.01 & 0.01 & < d.1. & 0.01 & 0.04 & 0.04 & 0.04 & 0.02 & 0.02 & 0.02 & $<$ d.1. & 0.02 & $<$ d.1. & 0.02 & 0.04 & 0.01 \\
\hline $\mathrm{Gd}$ & 0.13 & 0.07 & 0.01 & 0.04 & $<$ d.l. & $<$ d.l. & 0.09 & 0.15 & 0.14 & 0.06 & 0.07 & 0.07 & 0.02 & 0.13 & 0.02 & 0.07 & 0.15 & 0.04 \\
\hline $\mathrm{Tb}$ & 0.02 & 0.01 & $<$ d.1. & $<$ d.1. & < d.1. & $<$ d.1. & 0.01 & 0.02 & 0.02 & $<$ d.1. & $<$ d.l. & $<$ d.l. & $<$ d.l. & 0.02 & $<$ d.1. & 0.01 & 0.02 & 0.01 \\
\hline Dy & 0.11 & 0.08 & $<$ d.1. & $<$ d.l. & $<$ d.l. & $<$ d.1. & 0.08 & 0.13 & 0.12 & 0.02 & 0.03 & 0.02 & $<$ d.l. & 0.12 & 0.02 & 0.06 & 0.13 & 0.04 \\
\hline Ho & 0.02 & 0.01 & $<$ d.l. & $<$ d.1. & $<$ d.l. & $<$ d.1. & 0.01 & 0.02 & 0.02 & $<$ d.l. & 0.01 & $<$ d.l. & $<$ d.l. & 0.02 & $<$ d.1. & 0.01 & 0.02 & 0.01 \\
\hline $\mathrm{Er}$ & 0.05 & 0.03 & $<$ d.1. & $<$ d.1. & $<$ d.l. & $<$ d.1. & 0.04 & 0.06 & 0.06 & 0.01 & 0.01 & 0.01 & $<$ d.l. & 0.06 & 0.01 & 0.02 & 0.06 & 0.02 \\
\hline $\mathrm{Tm}$ & 0.02 & 0.02 & $<$ d.l. & $<$ d.l. & $<$ d.l. & $<$ d.1. & 0.02 & 0.02 & 0.02 & $<$ d.l. & $<$ d.l. & $<$ d.l. & $<$ d.l. & 0.01 & $<$ d.l. & 0.01 & 0.02 & 0.01 \\
\hline $\mathrm{Yb}$ & 0.05 & 0.03 & $<$ d.1. & $<$ d.1. & $<$ d.1. & $<$ d.1. & 0.03 & 0.06 & 0.06 & 0.01 & 0.01 & 0.01 & $<$ d.l. & 0.05 & 0.01 & 0.02 & 0.06 & 0.02 \\
\hline $\mathrm{Lu}$ & 0.02 & $<$ d.l. & $<$ d.l. & $<$ d.l. & $<$ d.l. & $<$ d.1. & $<$ d.l. & 0.02 & 0.02 & $<$ d.l. & $<$ d.l. & $<$ d.l. & $<$ d.l. & 0.02 & $<$ d.1. & 0.02 & 0.02 & 0.00 \\
\hline $\mathrm{Hf}$ & 0.49 & 0.63 & 0.47 & 0.57 & 0.48 & 0.42 & 0.56 & 0.51 & 0.56 & 0.34 & 0.37 & 0.35 & 0.32 & 0.64 & 0.56 & 0.48 & 0.64 & 0.09 \\
\hline $\mathrm{Ta}$ & $<$ d.1. & 0.05 & 0.02 & 0.04 & $<$ d.l. & $<$ d.1. & $<$ d.l. & 0.03 & 0.09 & $<$ d.l. & $<$ d.l. & $<$ d.1. & $<$ d.1. & 0.04 & $<$ d.1. & 0.04 & 0.10 & 0.03 \\
\hline W & 0.66 & 0.86 & 1.08 & 0.83 & 1.30 & 0.60 & 0.40 & 0.79 & 0.95 & 1.35 & 1.68 & 0.96 & 0.56 & 1.04 & 0.44 & 0.79 & 2.06 & 0.40 \\
\hline $\mathrm{Tl}$ & 0.14 & 0.07 & 0.13 & 0.28 & 0.39 & 0.12 & 0.08 & 0.18 & 0.37 & 0.33 & 0.34 & 0.34 & 0.02 & 0.13 & 0.18 & 0.17 & 0.39 & 0.11 \\
\hline $\mathrm{Pb}$ & 27.65 & 14.45 & 24.37 & 45.34 & 62.50 & 6.84 & 13.76 & 28.87 & 44.71 & 57.39 & 44.36 & 40.47 & 4.44 & 14.46 & 28.96 & 24.63 & 62.50 & 16.26 \\
\hline $\mathrm{Bi}$ & 0.51 & 0.25 & 0.64 & 0.96 & 1.38 & 0.24 & 0.73 & 0.55 & 0.95 & 1.65 & 0.99 & 0.97 & 0.19 & 0.41 & 0.74 & 0.57 & 1.65 & 0.36 \\
\hline Th & 0.17 & 0.09 & $<$ d.l. & 0.09 & $<$ d.1. & 0.05 & 0.04 & 0.23 & 0.22 & $<$ d.1. & $<$ d.1. & $<$ d.l. & $<$ d.l. & 0.29 & 0.08 & 0.13 & 0.29 & 0.07 \\
\hline $\mathrm{U}$ & 0.17 & 0.15 & $<$ d.l. & $<$ d.1. & $<$ d.l. & $<$ d.1. & 0.15 & 0.21 & 0.18 & 0.04 & 0.04 & 0.05 & $<$ d.l. & 0.19 & 0.06 & 0.10 & 0.21 & 0.06 \\
\hline \multicolumn{19}{|l|}{$\mu \mathrm{g} \mathrm{m}^{-3}$} \\
\hline Mineral & 13.8 & 5.1 & 10.3 & 11.6 & 7.7 & 8.5 & 8.2 & 16.5 & 14.6 & 7.8 & 9.4 & 8.7 & 3.1 & 15.7 & 3.2 & 8.3 & 16.5 & 3.2 \\
\hline $\mathrm{OM}+\mathrm{EC}$ & 7.0 & 6.9 & 11.2 & 11.0 & 11.8 & 4.5 & 4.3 & 8.7 & 10.4 & 9.0 & 18.2 & 13.4 & 5.1 & 11.7 & 9.1 & 9.0 & 18.2 & 3.1 \\
\hline SIC & 10.1 & 7.6 & 10.4 & 18.9 & 37.0 & 4.0 & 6.2 & 9.5 & 12.7 & 25.9 & 17.4 & 22.4 & 4.2 & 10.9 & 17.0 & 12.5 & 37.0 & 7.0 \\
\hline Marine & 1.4 & 0.6 & 0.8 & 0.7 & 1.0 & 1.6 & 2.1 & 0.7 & 0.6 & 1.3 & 1.7 & 1.7 & 1.1 & 0.8 & 0.6 & 1.1 & 2.3 & 0.4 \\
\hline Trace metals & 0.2 & 0.2 & 0.3 & 0.3 & 0.3 & 0.2 & 0.2 & 0.3 & 0.4 & 0.3 & 0.3 & 0.3 & 0.1 & 0.3 & 0.2 & 0.2 & 0.4 & 0.1 \\
\hline
\end{tabular}



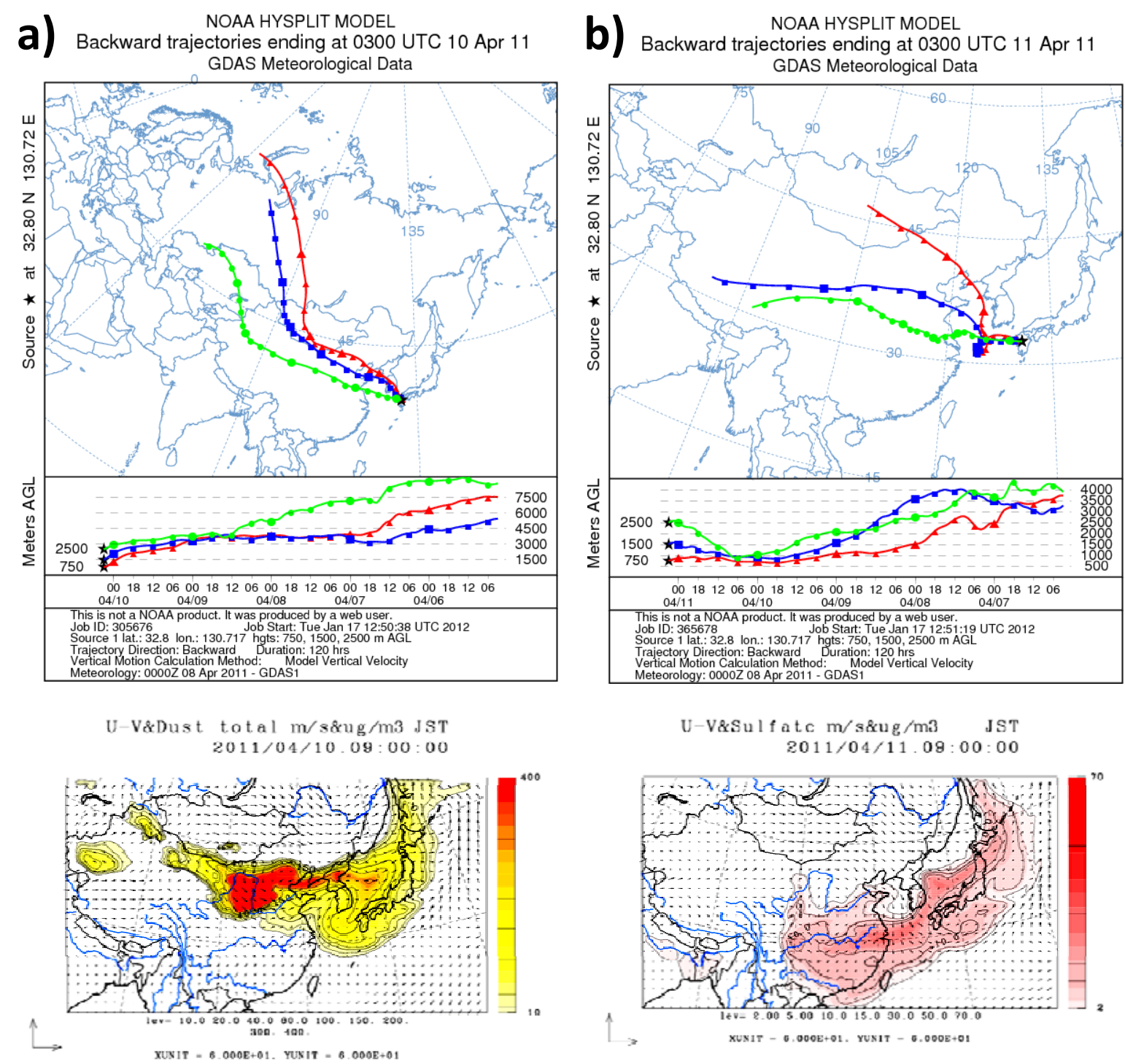

QRIAM/NIES

ORIAM/NIES

Fig. 3. Examples of HYSPLIT backtrajectories (top) and CFORS maps (bottom) illustrating the arrival of air masses from the Gobi Desert (a - 10 April 2011) and Asia mainland (b-11 April 2011) resulting in a dust (a) and a sulphate (b) event in Kumamoto.

was relatively uncontaminated, later peaks (220311, 240311 Table 1) coincide with peaks in $\mathrm{S}_{0.1-2.5}$ (Fig. $4 \mathrm{a}, \mathrm{c}$ ) and $\mathrm{SIC}\left(\mathrm{SO}_{4}^{2-}+\mathrm{NO}_{3}^{-}+\mathrm{NH}_{4}^{+}>10 \mu \mathrm{g} \mathrm{m}{ }^{-3}\right)$. The cleanest conditions were produced by rainfall (afternoon of $20 \mathrm{March}$ ), and five short-lived $\mathrm{NaCl}$ hourly peaks occurred during periods of increased wind speed blowing sea spray into the island (Fig. 4d). The passing waves of aerosols arriving into Kyushu from offshore were interspersed with periods of light winds and low temperatures when traffic-derived local pollutants produced high $\mathrm{NO}_{3}^{-}$relative to $\mathrm{SO}_{4}^{2-}$ and concentration spikes in several metals $(\mathrm{Cr}, \mathrm{Co}, \mathrm{Ni}, \mathrm{Cu}, \mathrm{Zn}, \mathrm{Sb})$ and carbon (240311 Table 1).

\subsection{Phase 2: 28 March-3 April}

During this phase a sulphatic transboundary intrusion, driven by an anticyclone initially persisting in east central China then moving east, degraded air quality over much of the NW Pacific (Fig. 1). Peak daily average $\mathrm{PM}_{10}$ concentrations at Kumamoto reached $66 \mu \mathrm{g} \mathrm{m}^{-3}$ (290311 Table 1), an increase mainly attributable to a sharp rise in SIC $\left(<10 \mu \mathrm{g} \mathrm{m}^{-3}\right.$ to $>20 \mu \mathrm{g} \mathrm{m}^{-3}$ ). On some days there were also increases in Gobi Desert dust as cold high level air sourced from Mongolia raised daily mineral dust levels back to $>10 \mu \mathrm{g} \mathrm{m}^{-3}$ (Fig. 4c, 280311 Table 1). Streaker data demonstrate these Kosa peaks to have been highly transient in nature, rising and 


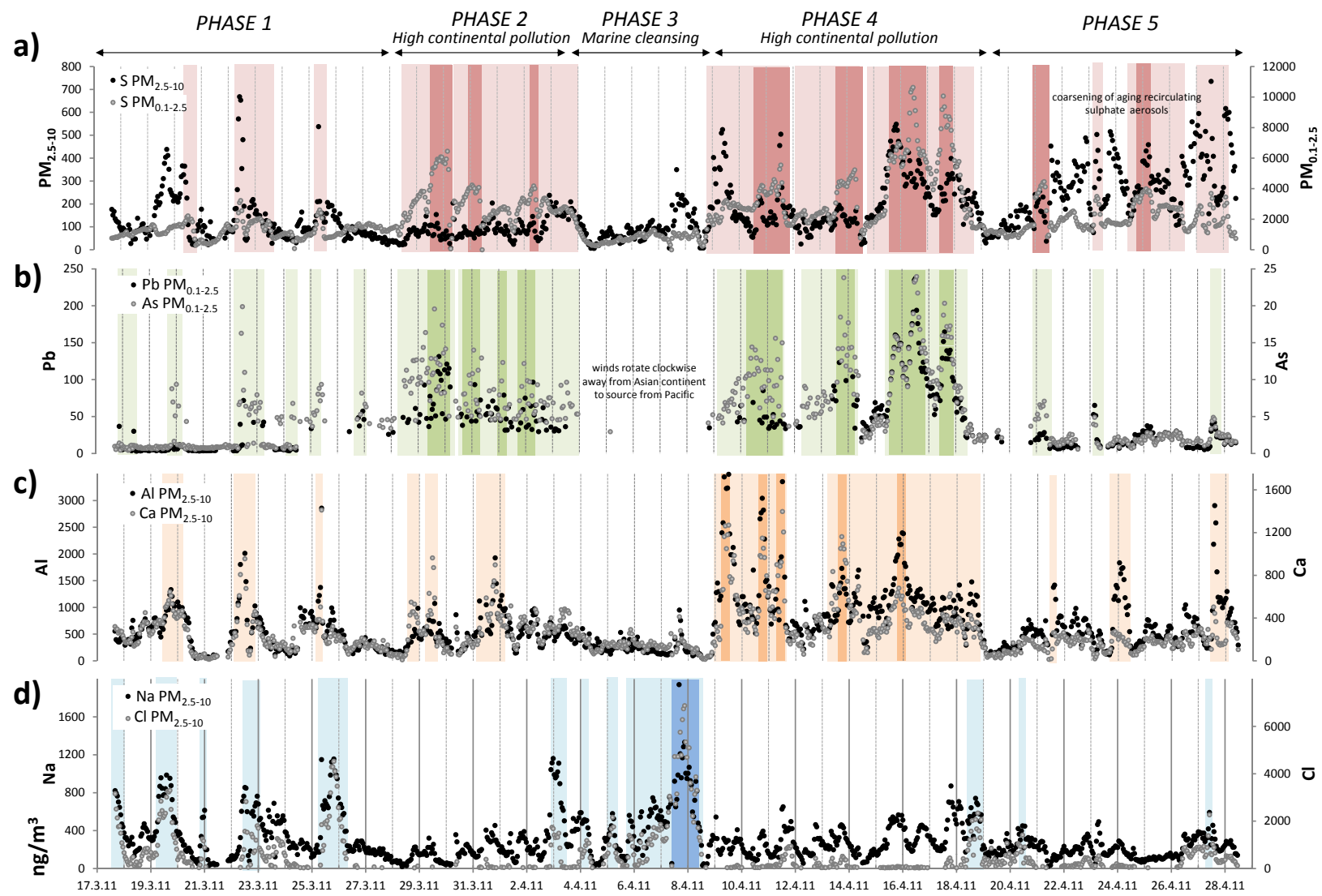

Fig. 4. Hourly selected elemental concentrations $\left(\mathrm{ng} \mathrm{m}^{-3}\right)$ obtained with the Streaker sampler for the five air quality phases identified during the monitoring campaign, with coloured bands highlighting main episodes of continental pollution and advection of salty marine air across Kumamoto. (a) shows both coarse $\left(\mathrm{PM}_{2.5-10}\right)$ and fine $\left(\mathrm{PM}_{0.1-2.5}\right)$ sulphatic aerosol intrusions, (b) uses $\mathrm{Pb}$ and As to illustrate how toxic metallic aerosols accompany the sulphatic intrusions, (c) uses Al and Ca to reveal the rise and fall of Gobi Desert PM introduced by NW winds crossing $\mathrm{NE}$ China, and (d) uses $\mathrm{Na}$ and $\mathrm{Cl}$ to identify marine aerosol episodes.

falling over half a day or less (Fig. 4c). In contrast, metalliferous sulphatic pollutants build up more gradually to successive peaks which tend to arrive slightly later than the "mineral dust" peak, and linger in the atmosphere as fine grained, atmospherically persistent particles (Fig. 4b). Another chemical characteristic of this phase is relatively high levels of $\mathrm{NO}_{3}^{-}$which over the first four days rise above $8.0 \mu \mathrm{g} \mathrm{m}{ }^{-3}$. Such concentrations are attributed to the mixing of cold, humid air with industrial pollutants, inhibiting thermal dissociation of $\mathrm{NH}_{4} \mathrm{NO}_{3}$ and so favouring high levels of particulate $\mathrm{NH}_{4} \mathrm{NO}_{3}$ which attained a campaign maximum on 2930 March 290311 Table 1) during cold $\left(<10^{\circ} \mathrm{C}\right)$, misty, and wet conditions in Kumamoto. Subsequent average daily temperatures rose into double figures and such elevated levels of $\mathrm{NO}_{3}^{-}$were not repeated.

\subsection{Phase 3: 3-8 April}

Advection of cleansing marine air across Kyushu removes the Asian mainland influence and results in a series of $\mathrm{NaCl}$ peaks recorded by the Streaker data (Fig. 4d; 080411 Ta- ble 1). Ambient $\mathrm{PM}_{10}$ levels fall to around $20 \mu \mathrm{g} \mathrm{m}^{-3}$ (samples 060411 and 070411, Table S1), and levels of contaminants $\mathrm{Ct}, \mathrm{NO}_{3}^{-}, \mathrm{SO}_{4}^{2-}, \mathrm{NH}_{4}^{+}, \mathrm{Sc}, \mathrm{V}, \mathrm{Ni}, \mathrm{Cu}, \mathrm{As}, \mathrm{Cd}, \mathrm{Sn}, \mathrm{Pb}$, and $\mathrm{Bi}$ all decline to a campaign minimum. In fact, by the end of this phase, concentrations of the most toxic elements such as $\mathrm{Pb}, \mathrm{Bi}, \mathrm{As}$, and $\mathrm{Cd}$ are a mere $5-8 \%$ of their maxima reached during Phase 2 the previous week. Ambient levels of mineral dust are subdued to within the narrow range of 5$8 \mu \mathrm{g} \mathrm{m}^{-3}$, with the exception of a transient peak on 7 April (Fig. 4c) which we attribute to a local resuspension event under unusually strong afternoon winds $\left(6 \mathrm{~ms}^{-1}\right)$. Light rain $\left(1-3 \mathrm{~mm} \mathrm{~h}^{-1}\right)$ was recorded only on the last day of phase 3 , the rest of the days being dry, including 6 April when the lowest PM concentration was recorded, indicating that rainfall did not influence the PM concentration during the arrival and cleansing of this oceanic advection event.

\subsection{Phase 4: 8-19 April}

A return to anticyclonic atmospheric stagnation over Central China creates a pollution plume which is joined from the NW 
by a Gobi dust intrusion and both move eastward into Japan (Fig. 3).The dilute southern edge of the $\mathrm{SO}_{4}^{2-}$ plume arrives in Kyushu on the afternoon of 8 April (Fig. 4a), followed that evening by Gobi dust which peaks the following midday (Fig. 4c). This is the biggest Kosa event in the campaign (sample 090411 Table 1, $45 \mu \mathrm{g} \mathrm{PM}_{10} \mathrm{~m}^{-3}$ ) and is followed by further mineral dust peaks over the next two days (Figs. 3a and 4c) as the dust intrusion recirculates over Kyushu producing campaign maxima in $\mathrm{Ca}, \mathrm{Al}, \mathrm{Fe}, \mathrm{K}, \mathrm{Mg}, \mathrm{Li}, \mathrm{Be}, \mathrm{Sc}$, Ti, Mn, Co, Rb, Sr, REE, Ta, U (samples 090411, 100411 Table 1). Sulphur $\mathrm{PM}_{0.1-2.5}$ recorded by the Streaker also peaks at midday 9 April but then does not decline significantly (unlike the mineral dust: Fig. 4a, c).

The final part of this prolonged regional pollution event was especially interesting. Whereas all previous arrivals of SIC contaminants had travelled out from China via a NW corridor typically involving transport over the Yellow Sea, by 15 April a concentrated wave of industrial pollutants was travelling directly east from the Shanghai coast into western Japan. This fresh new wave of transboundary aerosols produced the most pronounced pollution event during the campaign $\left(18 \mu \mathrm{g} \mathrm{SO}_{4}^{2-} \mathrm{m}^{-3}\right)$. Beginning with the arrival around midnight on 15 April of a brief peak of aluminous dust accompanied by SIC (Fig. 4a, c), levels of anthropogenic contaminants were to hit new maxima on 16 and 17 April (Fig. 4b; samples 150411, 160411, 170411 Table 1). The trace element content accompanying this sulphatic and carbonaceous aerosol intrusion is again very metalliferous (high $\mathrm{V}, \mathrm{Cd}, \mathrm{Sn}, \mathrm{Bi}, \mathrm{K}, \mathrm{Pb}, \mathrm{Sb}, \mathrm{As}, \mathrm{Zn}, \mathrm{W}$ ) but the chemical mix is subtly different from those recorded previously, with unusually high levels of total carbon, $\mathrm{V}, \mathrm{Cd}, \mathrm{Sn}$ and differences in element ratios (e.g. higher As/Se). This multiple pollution event was finally terminated on the evening of 18 April with the arrival of strong, clean NNW winds carrying enough sea spray to mark a marine event (Fig. 4d).

\subsection{Phase 5: 19-28 April}

The northerly advection event which swept the Phase 4 pollution plume south of Japan during 18-19 April was initially accompanied by an uncontaminated Kosa event which briefly raised crustal dust levels as SIC concentrations continued to fall rapidly (compare Fig. 4a and c, 18 April). The following day, still dominated by a strong northerly Russian airflow moving into Kyushu via the Korean Peninsula and Sea of Japan, provided the cleanest $24 \mathrm{~h}$ filter of the entire sampling campaign, with $\mathrm{PM}_{10}$ levels dropping to $18.6 \mu \mathrm{g} \mathrm{m}^{-3}$, mineral dust to $3 \mu \mathrm{g} \mathrm{m}^{-3}$ and SIC to $4 \mu \mathrm{g} \mathrm{m}^{-3}$ (190411 Table 1). However this rapid and thorough atmospheric cleansing event provided only brief respite from the Chinese pollution plume, which continued to recirculate widely across the NW Pacific and returned into Japan on 20 April. For the remainder of the campaign this diluted but persistent $\mathrm{SO}_{4}^{2-}$ haze recirculated over Japan, occasionally supplemented by influxes of fresh mainland contaminants and Gobi dust to produce several minor $\mathrm{S}_{0.1-2.5}$ and $\mathrm{Al}_{2.5-10}$ peaks (Fig. 4a and 4c; 240411 Table 1). Comparison between the coarser and finer PM fractions in the Streaker data indicate an increase in the coarser sulphate particles $\left(\mathrm{S}_{\mathrm{PM}_{0.1-2.5} / \mathrm{PM}_{2.5-10}}\right.$ drops from 20.4 in Phase 4 to 9.9 in Phase 5), suggesting a relative coarsening by coagulation and chemical interaction of PM with gaseous precursors with time as the regional pollution plume ages and persists across the NW Pacific region (Fig. 4a).

\section{Discussion and conclusions}

The chemical data summarized in this paper allow us to view both hourly and daily fluctuations in transboundary aerosols affecting Japanese ambient air quality over a 6-week period in spring, and provide a clearer idea of the reality of what people are inhaling at that time of the year. Such data highlight the limitations of legal air quality standards based only on PM mass without considering the complex chemical mixture of inhalable aerosols involved.

The PMF analysis of Streaker hourly data (Fig. 4) confirms that natural PM predominates in the coarser PM fraction $\left(\mathrm{PM}_{2.5-10}\right)$, with continental mineral dust dominant over fresh marine aerosol. In the finer fraction $\left(\mathrm{PM}_{0.1-2.5}\right)$ however the mineral dust is much less abundant and instead the dominant component is As-bearing sulphatic aerosol. Another type of sulphatic aerosol (rich in $\mathrm{Zn}$ and $\mathrm{Cu}$ ) has been recognized in the finer fraction. Although $\mathrm{As}, \mathrm{Zn}$, and $\mathrm{Cu}$ all could result from coal combustion (Okuda et al., 2008), our PMF analysis suggests that As and the other metals have different sources. The metalliferous sodic PM component recognised in both size fractions is suggested to represent dechlorinated, sulphate-enriched aged sea salt aerosols contaminated by industrial emissions during long-distance transport. In both size fractions local PM sources, such as traffic, are relatively minor. Sea-salt particles may play a role in scavenging metalliferous components in the atmosphere, like the way they increase the settling velocity of dust particles by adhering and enhancing the particle size (Zhang, 2008). Concentration of $\mathrm{Zn}$ in $\mathrm{PM}_{10}$ in Kumamoto (up to $151 \mathrm{ng} \mathrm{m}^{-3}$, Table 1) is about a half to one fourth of that reported from Korea during Asian dust events (up to $590 \mathrm{ng} \mathrm{m}^{-3}$, Park and Dam, 2010).

No extreme Kosa events (when average daily $\mathrm{PM}_{10}$ concentrations in Japan can rise dramatically to well above $100 \mu \mathrm{g} \mathrm{m}^{-3}$ ) were recorded during the campaign (in fact the year 2011 had almost one third less Kosa events than the previous year according to the Japan Meteorological Agency on Kosa observations, http://www.data.kishou.go.jp/ obs-env/kosahp/kosa_table_1.html), but instead our sampling interval was typical of what these days may be considered normal springtime conditions. Under such conditions prevailing NW winds bring frequent but normally brief intrusions of Gobi dust into western Japan so that during the 
Correlation STREAKER chemical data/CFORS model

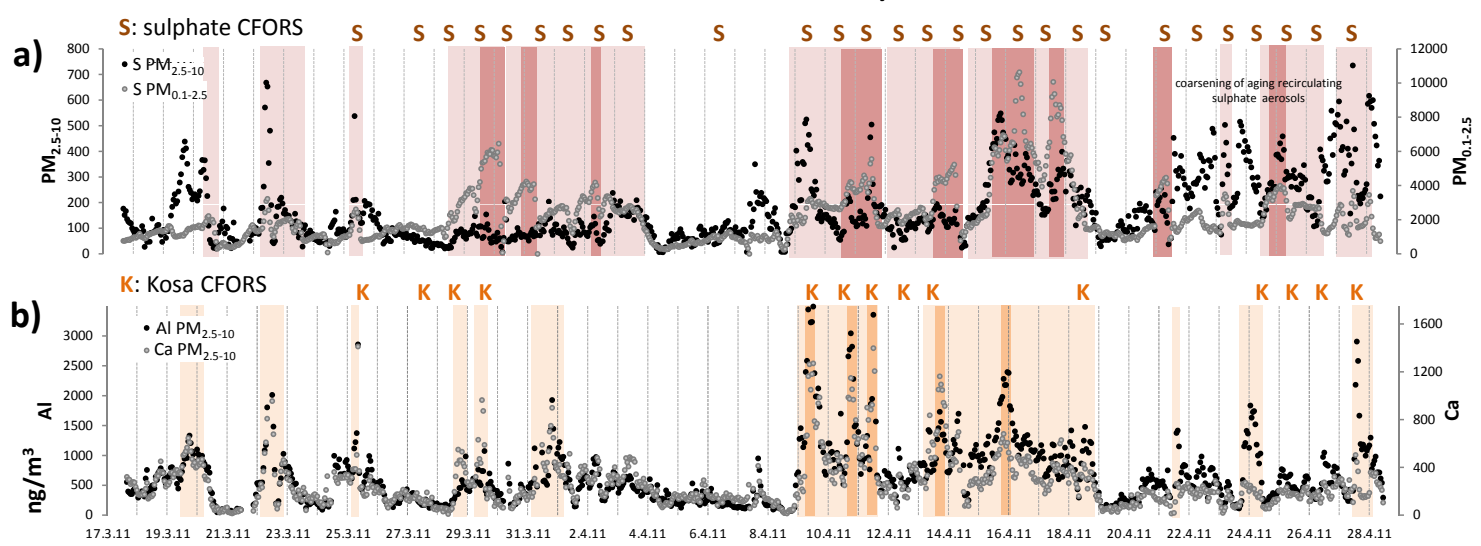

Fig. 5. Hourly Streaker data for sulphatic peaks (a) and mineral dust peaks (b). Days when sulphatic (S) and dust (K) events were predicted by the Chemical Weather Forecasting System (CFORS).

combined 4-week period of phases 1,2 and 5 our data recorded nine such "natural" events when concentrations of $\mathrm{Al}$ exceeded $1 \mu \mathrm{g} \mathrm{m}^{-3}$ (Fig. 4c) for a few hours. A notably heavier loading of mineral dust occurred during the pollution episode of Phase 4 , but even here the peaks remained highly transient, with concentrations of $\mathrm{Al}$ rising from around $1 \mu \mathrm{g} \mathrm{m}^{-3}$ to double or triple over 2 or $3 \mathrm{~h}$ but then falling back equally rapidly. In contrast the anthropogenic component of suspended particulate matter in the atmosphere usually builds up more slowly and does not fully decline as rapidly as the mineral dust. Once $\mathrm{S}_{0.1-2.5}$ levels rose above $2 \mu \mathrm{g} \mathrm{m}^{-3}$ during the pollution episodes of Phases 2 and 4 they stayed high, reflecting the atmospherically persistent nature of this extremely fine sulphatic PM. The same behaviour can be seen in the metalliferous components, well displayed by $\mathrm{Pb}, \mathrm{Zn}, \mathrm{Sn}$ and As on Fig. 4a-b which consistently correlate more with the sulphatic component than with the natural mineral dust. Sulphatic plumes dissipate only slowly, due to the dominance of submicron accumulation mode aerosols which are atmospherically persistent, although interestingly our Streaker data for Phase 5 suggest that the toxic metal loading may decrease as the airmass ages and coarsens by coagulation (Fig. 4a, b).

The arrival of contaminated air masses from mainland Asia is forecasted daily by the Chemical Weather Forecasting System (CFORS) which calculates distributions of Asian dust and anthropogenic sulphate aerosols every three hours, the results being uploaded on the website every day (http://www-cfors.nies.go.jp/ cfors/; Fig. 3). Our database has enabled us to compare hourly ground observations (using the Streaker) with those predicted by CFORS. At the start of our sampling campaign CFORS data were unavailable due to the disruption caused by the Tōhoku Earthquake on 11 March 2011. The system was reinstated on 25 March, however, so that we were able to follow CFORS predictions for the remaining 34 days of the campaign. During this pe- riod there were only four days when CFORS $\mathrm{SO}_{4}$ predictions failed to correspond with our chemical data collected on the ground. In all four cases sulphate pollution was predicted but not recorded specifically at that time, this being due either to slightly delayed arrival (27 March) or more rapid passing (11 and 19 April) of the sulphate cloud, or a "featheredge effect" when the predicted margin of the polluted airmass just missed the Kumamoto area (6 April). Figure 5a superimposes on the Streaker data the days when $\mathrm{SO}_{4}$ transboundary intrusions (S) were forecast by CFORS, and demonstrates that CFORS is in general an excellent predictor of transboundary sulphatic air masses.

In the case of transboundary "crustal" dust events our data demonstrate that not all silicate dust peaks can be attributed directly to Kosa events forecast by CFORS. During our sampling campaign CFORS predicted the presence of Kosa dust intrusions on 14 separate days (marked as K on Fig. 5b), and all of these were detectable on the ground as transient $\mathrm{CaAl}$ peaks in our Streaker dataset (with the one exception of the 27 March delayed arrival also noted for $\mathrm{SO}_{4}$ ). There were, however, three episodes when transient $\mathrm{CaAl}$ peaks occurred despite the fact that no Kosa event had been forecast. In all three cases these episodes occurred during the major sulphatic events. This observation indicates that the atmospherically persistent sulphatic PM cloud remains chemically heterogeneous, containing areas rich in Al silicates (presumably some combination of natural minerals and flyash), the passing of which are recorded as transient peaks by the Streaker data (for example see 3 March, 16 and 21 April on Fig. 4c).

It is clear that transboundary aerosol intrusions arriving into Japan are chemically inhomogeneous. As we have observed in our precursor pilot study of Kosa events in Kyushu, some waves of Gobi dust are more calcareous than others, presumably depending on their geological source area (Yuan et al., 2008; Zhang et al., 2005; Shao et al., 2008; Moreno et al., 2012). The Streaker data reinforce this observation of 
natural chemical variables in transboundary events, with later mineral dust peaks in phases 4 and 5 registering as more aluminous than earlier, more calcareous ones (Fig. 4c). Furthermore, our data reveal distinct differences in the chemical signature of the Asiatic mainland pollution plume, even after a journey of around $1000 \mathrm{~km}$ or more from source. The best example is provided by the unusually high levels of $\mathrm{SO}_{4}^{2-}$, $\mathrm{V}, \mathrm{Cd}, \mathrm{Sn}, \mathrm{Bi}, \mathrm{Cu}, \mathrm{As}, \mathrm{Sb}$, and $\mathrm{Pb}$ in response to the arrival of pollution directly from East China into Kyushu on 15 April. Yet another confounding factor for those trying to determine the possible health effects of transboundary aerosols is the way peak concentration arrivals of mineral dust are commonly not synchronous with anthropogenic pollution peaks. A good example of this is provided by Phase 4 when the initial arrival of a Kosa PM wave was accompanied by a much slower build up to a sulphate peak (Fig. 4a and c). Indeed, the high levels of natural dust at the beginning of Phase 4 contrast greatly with the later peaks in anthropogenic contaminants as the sulphatic plume was repeatedly recharged by mainland-sourced pollution without any additional influence of Gobi Desert dust. Finally, while still on the theme of chemical variation within the East Asian pollution plume, our data show considerable differences in ambient PM nitrate concentration (from 1 to $16 \mu \mathrm{g} \mathrm{m}^{-3}$ ), depending not just on local versus exotic sources but also on temperature controlling the volatility of atmospheric ammonium nitrate.

The magnitude of the loss in air quality over western Japan due to the 21st century spread of the mainland Asiatic pollution plume is emphasized by our Phase 3 record of 5 days when winds brought air from the oceanic rather than continental sector. As the origin of these winds crossing SW Japan rotated clockwise from north to east to south we see proof of how little regional industrial air pollution these days originates from the Japanese archipelago. Average levels of anthropogenic pollutants elements fall abruptly, in some cases by $>90 \%$, as the continental source is removed. This is especially true of the most undesirable pollutants: $\mathrm{Pb}$ from $>60$ to $3 \mathrm{ng} \mathrm{m}^{-3}$, As from 6 to $0.3 \mathrm{ng} \mathrm{m}^{-3}$, Cd from 1.1 to $0.09 \mathrm{ng} \mathrm{m}^{-3}$.

Although the concentrations are lower than in many parts of urban China and Korea (Kan et al., 2007; Okuda et al., 2008; Zhao et al., 2008; Saikawa et al., 2009; Park and Dam, 2010; Zheng et al., 2011), their atmospheric persistence and highly respirable nature create reasonable cause for concern. Much of this concern has focussed more on the pulmonary toxicity and corresponding acute effects of PM inhalation, especially on asthmatic patients (e.g. Ichinose et al., 2005; Ueda et al., 2010; Watanabe et al., 2011), or on the potential transport of active bioaerosols between countries (e.g. Chen et al., 2010). However, a greater health problem is more likely to be related to premature deaths from cardiovascular and respiratory diseases resulting from long-term exposure to elevated levels of atmospherically persistent, respirable, metalliferous PM (Chan et al., 2008; Liu et al., 2009).
The main conclusions of the present study can be summarized as follows:

1. Based on the PMF analysis, the coarser PM fraction $\left(\mathrm{PM}_{2.5-10}\right)$ is dominated by mineral dust and sea salt, whereas the finer fraction $\left(\mathrm{PM}_{0.1-2.5}\right)$ is largely sulphate that includes As-bearing and $\mathrm{Zn}$-Cu-bearing particles. The PMF results also suggest that metals can be incorporated into aged sea salt.

2. Mineral dust arrivals are highly transient, usually peaking over a few hours before rapidly declining. This contrasts with the more atmospherically persistent sulphatic transboundary aerosols, although these may decline in their toxic metal content as they age.

3. CFORS is an excellent predictor for transboundary sulphatic PM intrusions. However, our hourly monitoring detected a few CaAlSi mineral dust peaks that were not predicted by CFORS. These transient mineral dust peaks all occurred during major sulphatic events indicating an atmospherically persistent but poorly mixed sulphatic $\mathrm{PM}$ cloud containing areas rich in $\mathrm{Al}$ silicates.

4. Transboundary aerosol plumes in Eastern Asia can be highly chemically inhomogeneous, depending on the geology of the source area (e.g. calcareous or not), the nature of the pollutants they incorporate during their generation (producing contrasting mixtures of metalliferous PM), and the amount of mixing during the varied physico-chemical conditions they encounter during transport (nitrate and sea salt content).

5. The dominant influence on air quality at Kumamoto, and by analogy probably across much of the Japanese Archipelago, is the regular arrival of air masses contaminated with sulphate and toxic metals and sourced from mainland Asia. The contrast between atmospheric conditions under westerly and easterly airflows crossing Japan is striking and a cause for concern with regard to human health effects.

\section{Supplementary material related to this article is available online at: http://www.atmos-chem-phys.net/13/ 1411/2013/acp-13-1411-2013-supplement.pdf.}

Acknowledgements. This work was supported by the Invitation Fellowship Program for Research of the Japan Society for the Promotion of Science (No. 11019, TM), by the Generalitat de Catalunya (CUR-DIUE: BE-DGR 2010, TM), and partly by the Grant-in-Aid for Scientific Research (No. 17684026, TK). TK also thanks D. Zhang and T. Nagatani for cooperation with air monitoring in the Kumamoto area.

Edited by: E. Gerasopoulos 


\section{References}

Aikawa, M., Ohara, T., Hiraki, T., Oishi, O., Tsuji, A., Yamagami, M., Murano, K., and Mukai, H.: Significant geographic gradients in particulate sulfate over Japan determined from multiple-site measurements and a chemical transport model: impacts of transboundary pollution from the Asian continent, Atmos. Environ., 44, 381-391, 2010.

Amato, F., Pandolfi, M., Escrig, A., Querol, X., Alastuey, A., Pey, J., Perez, N., and Hopke, P. K.: Quantifying road dust resuspension in urban environment by multilinear engine: a comparison with PMF2, Atmos. Environ., 43, 2770-2780, 2009.

Arimoto, R., Zhang, X. Y., Huebert, B. J., Kang, C. H., Savoie, D. L., Prospero, J. M., Sage, S. K., Schloesslin, C. A., Khaing H. M., and Oh, S. N.: Chemical composition of atmospheric aerosols from Zhenbeitai China, and Gosan, South Korea, during ACE-Asia, J. Geophys. Res., 109, D19So4, doi:10.1029/2003JD004323, 2004.

Birch, M. E. and Cary, R. A.: Elemental carbon based method for monitoring occupational exposures to particulate diesel exhaust, Aerosol Sci. Technol., 25, 221-241, 1996.

Chan, C. and Yao, X.: Air pollution in mega cities in China, Atmos. Environ., 42, 1-42, 2008.

Chan, C. C., Chuang, K. J., Chen, W. J., Chang, W. T., Lee, C. T., and Peng, C. M.: Increasing cardiopulmonary emergency visits by long-range transported Asian dust storms in Taiwan, Environ. Res., 106, 393-400, 2008.

Chen, C., Tsa, F., Lin, C., Yang, C., Chan, C., Young, C., and Lee, C.: Ambient influenza and avian influenza virus during dust storm days and background days, Environ. Health Perspect., 118, 1211-1216, 2010.

Cheng, M. T., Lin, I. C., Chio, C. P., Wang, C. F., and Kuo, C. Y.: Characteristics of aerosols collected in central Taiwan during an Asian dust event in spring 2000, Chemosphere, 61, 1439-1450, 2005.

Chung, Y. S. and Kim, H. S.: Observations of massive air-pollution transport and associated air quality in the Yellow Sea region, Air Qual. Atmos. Health, 1, 69-79, 2008.

Draxler, R. R. and Rolph, G. D.:HYSPLIT (HYbridSingle-Particle Lagrangian Integrated Trajectory) Model Access via NOAA ARL READY Website, NOAA Air Resources Laboratory, SilverSpring, MD, http://www.arl.noaa.gov/ready/hysplit4.html, 2003.

Fairlie, T. D., Jacob, D. J., Dibb, J. E., Alexander, B., Avery, M. A., van Donkelaar, A., and Zhang, L.: Impact of mineral dust on nitrate, sulfate, and ozone in transpacific Asian pollution plumes, Atmos. Chem. Phys., 10, 3999-4012, doi:10.5194/acp-10-39992010, 2010.

Guo, J., Rahn, K., and Zhuang, G.: A mechanism for the increase of pollution elements in dust storms in Beijing, Atmos. Environ., 38, 855-862, 2004.

Ichinose, T., Nishikawa, M., Takano, H., Sera, N., Sadakane, K., Mori, I., Yanagisawa, R., Oda, T., Tamura, H., Hiyoshi, K., Quan, H., Tomura, S., and Shibamoto T.: Pulmonary toxicity induced by intratracheal instillation of Asian yellow dust (Kosa) in mice, Environ. Toxicol. Pharmacol., 20, 48-56, 2005.

Kan, H .D., London, S., Chen, G., Zhang, Y., Song, G., Zhao, N., Jiang, L., and Chen, B.: Differentiating the effects of the fine and coarse particles on daily mortality in Shanghai, China, Environ. Int., 33, 376-384, 2007.
Kaneyasu, N., Takami, A., Sato, K., Hatakeyama, S., Hayashi, M., Hara, K., Kawamoto, K., and Yamamoto, S.: Year-round behavior of $\mathrm{PM}_{2.5}$ in a remote island and urban sites in the northern Kyushu area, Japan, Journal of Japan Society for Atmos. Environ., 46, 111-117, 2011.

Kim, J., Yoon, S., Jefferson, A., Zahorowski, W., and Kang, C: Air mass characterization and source region analysis for the Gosan super-site, Korea, during the ACE-Asia 2001 field campaign, Atmos. Environ., 39, 6513-6523, 2005.

Kim, K. H., Choi, G., Kang, C., Lee, J., Kim, J. Y., Youn, Y. H., and Lee, S.. R: The chemical composition of the fine and coarse particles in relation with the Asian dusts events, Atmos. Environ., 37, 753-765, 2003.

Kim, K. H., Mam, C. J., and Okuda, T: Analysis of long-term changes in airborne toxic metals in South Korea's two largest cities from 1991 to 2004, Environ, Sci. Poll. Res., 16, 565-572, 2009.

Kojima, T., Buseck, P., Iwasaka, Y., Matsui, A., and Tronchkine, D: Sulfate-coated dust particles in the free troposphere over Japan, Atmos. Res., 6, 698-708, 2006.

Kong, S., Han, B., Bai, Z., Chen, L., Shi, J., and Xu, Z: Receptor modeling of $\mathrm{PM}_{2.5}, \mathrm{PM}_{10}$ and TSP in different seasons and longrange transport analysis at a coastal site of Tianjin, China, Sci. Total Environ., 408, 4681-4694, 2010.

Lasserre, F., Cautenet, G., Bouet, C., Dong, X., Kim, Y. J., Sugimoto, N., Matsui, I., and Shimizu, A.: A model tool for assessing real-time mixing of mineral and anthropogenic pollutants in East Asia: a case study of April 2005, Atmos. Chem. Phys., 8, 36033622, doi:10.5194/acp-8-3603-2008, 2008.

Liu, J. and Mauzerall, D. L.: Potential influence of inter-continental transport of sulfate aerosols on air quality, Environ. Res. Lett., 2, 045029, doi:10.1088/1748-9326/2/4/045029, 2007.

Liu, J., Mauzerall, D. L., and Horowitz, L. W.: Source-receptor relationships between East Asian sulfur dioxide emissions and Northern Hemisphere sulfate concentrations, Atmos. Chem. Phys., 8, 3721-3733, doi:10.5194/acp-8-3721-2008, 2008.

Liu, J., Mauzerall, D. L., and Horowitz, L. W.: Evaluating intercontinental transport of fine aerosols: (2) Global health impact, Atmos. Environ., 43, 4339-4347, 2009.

Lucarelli, F., Nava, S., Calzolai, G., Chiari, M., Udisti, R., and Marino, F.: Is PIXE still a useful technique for the analysis of atmospheric aerosols? The LABEC experience, X-Ray Spectrometry, 40, 162-167, 2011.

Ma, C., Tohno, S., Kasashara, M., and Hayakawa, S.: The nature of individual solid particles retained in size-resolved raindrops fallen in Asian dust store event during ACE-Asia, Atmos. Environ., 38, 2951-2964, 2004.

Moreno, T., Kojima, T., Querol, X., Alastuey, A., Amato, F., and Gibbons, W.: Natural versus anthropogenic inhalable aerosol chemistry of transboundary East Asian atmospheric outflows into western Japan, Sci. Total Environ., 424, 182-192, 2012.

Mori, I., Nishikawa, M., Tanimura, T., and Quan, H.: Change in size distribution and chemical composition of kosa (Asian dust) aerosol during long-range transport, Atmos. Environ., 37, 42534263, 2003.

Nagatani, T., Yamada, M., Kojima, T., and Zhang, D.: Episodic particulate sulfate and sulfur dioxide on the southwestern Japan coast in March and April 2010, Asian Journal of Atmos. Environ., 6, 41-52, 2012. 
Nishikawa, M., Kanamori, S., Kanamori, N., and Mizoguchi, T.: Kosa aerosol as eolian carrier of anthropogenic material, Sci. Total Environ., 107, 13-27, 1991.

Ohara, T., Akimoto, H., Kurokawa, J., Horii, N., Yamaji, K., Yan, X., and Hayasaka, T.: An Asian emission inventory of anthropogenic emission sources for the period 1980-2020, Atmos. Chem. Phys., 7, 4419-4444, doi:10.5194/acp-7-4419-2007, 2007.

Okuda, T., Katsuno, M., Naoi, D., Nakao, S., Tanaka, S., He, K., Ma, Y., Lei, Y., and Jia, Y.: Trends in hazardous trace metal concentrations in aerosols collected in Beijing, China from 2001 to 2006, Chemosphere, 72, 917-924, 2008.

Onishi, K., Kurosaki, Y., Otani, S., Yoshida, A., Sugimoto, N., and Kurozawa, Y.: Atmospheric transport route determines components of Asian dust and health effects in Japan, Atmos. Environ., 49, 94-102, 2012.

Paatero, P. and Hopke, P. K.: Discarding or downweighting highnoise variables in factor analytic models, Anal. Chim. Acta, 490, 277-289, 2003

Paatero, P. and Tapper, U.: Positive matrix factorization: a nonnegative factor model with optimal utilization of error estimates of data values, Environmetrics, 5, 111-126, 1994.

Park, K. and Dam, H.-D.: Characterization of metal aerosols in $\mathrm{PM}_{10}$ from urban, industrial, and Asian Dust sources, Environ. Monit. Assess., 160, 289-300, 2010.

Park, S., Bae, M., Schauer, J., Ryu, S., Kim, Y., Cho, S., and Kim, S: Evaluation of the TMO and TOT methods from OC and EC measurements and their characteristics in $\mathrm{PM}_{2.5}$ at an urban site of Korea during ACE-Asia, Atmos. Environ., 39, 5101-5112, 2005.

Prospero, J. M., Savoir, D. L., and Arimoto, R.: Long-term record of nss-sulfate and nitrate in aerosols on Midway Island, 1981-2000: evidence of increased (now decreasing?) anthropogenic emissions from Asia, J. Geophys. Res., 108, 4019, doi:10.1029/2001JD001524, 2003.

Querol, X., Alastuey, A., Rodríguez, S., Plana, F., Ruiz, C. R., Cots, N., Massagué, G., and Puig, O.: $\mathrm{PM}_{10}$ and $\mathrm{PM}_{2.5}$ source apportionment in the Barcelona Metropolitan Area, Catalonia, Spain, Atmos. Environ., 35/36, 6407-6419, 2001.

Saikawa, E., Naik, V., Horowitz, L., Liu, J., and Mauzerall, D.: Present and potential future contributions of sulphate, black \& organic carbon aerosols from China to global air quality, premature mortality and radiative forcing, Atmos. Environ., 43, 2814 $2822,2009$.

Satake, S., Uno, I., Takemura, T., Carmichael, G., Tang, Y., Streets, D., Sugimoto, N., Shimizu, A., Uematsu, M., Han, J.-S., and Ohta, S.: Characteristics of Asian aerosol transport simulated with a regional-scale chemical transport model during the ACE-Asia observation, J. Geophys. Res., 109, D19S22, doi:10.1029/2003JD003997, 2004.

Seinfeld, J., Carmichael, G., Arimoto, R., Conant, W., Brechtel, F., Bates, T., Cahill, T., Clarke, A., Doherty, S., Flatau, P., Huebert, B., Kim, J., Markowicz, K., Quinn, P., Russell, L., Russell, P., Shimizu, A., Shinozuka, Y., Song,Ch., Tang, Y., Uno, I., Vogelmann, A., Weber, R., Woo, J., and Zhang, X.: ACE-ASIA Regional Climatic and Atmospheric Chemical Effects of Asian Dust and Pollution, American Meteorological Society March 2004, doi:10.1175/BAMS-85-3-367, 2004.

Shao, L. Y., Li, W. J., Xiao, Z. H., and Sun, Z. Q.: The mineralogy and possible sources of spring dust particles over Beijing, Adv.
Atmos. Sci., 25, 395-403, 2008.

Shimohara, T., Osih, O., Utsunomiya, A., Mukai, H., Hatakeyama, S., Eun-Suk, J., Uno, I., and Murano, K.: Characterization of atmospheric air pollutants at two sites in northern Kyushu, Japan - chemical form, and chemical reaction, Atmos. Environ., 35, 667-681, 2001.

Takahashi, H., Naoe, H., Igarashi, Y., Inomata, Y., and Sugimoto, N.: Aerosol concentrations observed at Mt. Haruna, Japan, in relation to long-range transport of Asian mineral dust aerosols, Atmos. Environ., 44, 4638-4644, 2010.

Tobo, Y., Zhang, D., Nakata, N., Yamada, M., Ogata, H., Hara, K., and Iwasaka, Y: Hygroscopic mineral dust particles as influenced by chlorine chemistry in the marine troposphere, Geophys. Lett., 36, L05817, doi:10.1029/2008GL036883, 2009.

Trochkine, D., Iwasaka, Y., Matsuki, A., Yamada, M., Kim, Y. S., Nagatani, T., Zhang, D., Shi, G.-Y., and Shen, Z.: Mineral aerosol particles collected in Dunhuang, China, and their comparison with chemically modified particles collected over Japan, J. Geophys. Res., 108, 8642, doi:10.1029/2002JD003268, 2003.

Turpin, B. J., Saxena, P., and Andrews, E.: Measuring and simulating particulate organics in the atmosphere: problems and prospects, Atmos. Environ., 34, 2983-3013, 2000.

Ueda, K., Nitta, H., and Odajima, H.: The effects of weather, air pollutants, and Asian dust on hospitalization for asthma in Fukuoka Environ. Health Preventive Med., 15, 350-357, 2010.

Uno, I., Carmichael, G., Streets, D., Tang, Y., Yienger, J., Satake, S., Wang, Z., Woo, J., Guttikunda, S., Uematsu, M., Matsumoto, K., Tanimoto, H., Yoshioka, K., and Iida, T.: Regional chemical weather forecasting system CFORS: Model descriptions and analysis of surface observations at Japanese island stations during the ACE-Asia experiment, J. Geophys. Res., 108, 8668, doi:10.1029/2002JD002845, 2003.

Uno, I., Satake, S., Carmichael, G., Tang, Y., Wang, Z., Takemura, T., Sugimoto, N., Shimizu, A., Murayama, T., Cahill, T., Cliff, S., Uematsu, M., Ohta, S., Quinn, P., and Bates, T: Numerical study of Asian dust transport during the springtime of 2001 simulated with the chemical weather forecasting system (CFORS) model, J. Geophys. Res., 109, D19S24, doi:10.1029/2003JD004222, 2004.

Uno, I., Uematsu, M., Hara, Y., He, Y. J., Ohara, T., Mori, A., Kamaya, T., Murano, K., Sadanaga, Y., and Bandow, H.: Numerical study of the atmospheric input of anthropogenic total nitrate to the marginal seas in the westerns North Pacific region, Geophys. Lett., 34, L17817, doi:10.1029/2007GL030338, 2007.

Wang, X., Dong, Z., Zhang, J., and Liu, L.: Modern dust storms in China: an overview, J. Arid Environ., 58, 559-574, 2004.

Watanabe, M., Yamasaki, A., Burioka, N., Kurai, J., Moneda, K., Yoshida, A., Igishi, T., Fukuoka, Y., Nakamoto, M., Takeuchi, H., Suyama, H., Tatsukawa, T., Chikumi, H., Matsumoto, S., Sako, T., Hasegawa, Y., Okazaki, R., Horasaki, K., and Shimizu, E.: Correlation between Asian dust storms and worsening asthma in western Japan, Allergology International, 60, 267-275, 2011.

Yuan, H., Zhuang, G., Li, J., Wang, Z., and Li, J.: Mixing of mineral with pollution aerosols in dust season in Beijing: revealed by source apportionment study, Atmos. Environ., 42, 2141-2157, 2008.

Zhang, D.: Effect of sea salt on dust settling to the ocean, Tellus B, 60, 641-646, 2008.

Zhang, D., Iwasaka, Y., Shi, G., Zang, J., Matsuki, A., and Trochkine, D.: Mixture state and size of Asian dust particles col- 
lected at southwestern Japan in spring 2000, J. Geophys. Res., 108, 4760, doi:10.1029/2003JD003869, 2003.

Zhang, D., Iwasaka, Y., Matsuki, A., Ueno, K., and Matsuzaki, T.: Coarse and accumulation mode particles associated with Asian dust in southwestern Japan, Atmos. Environ., 40, 1205-1215, 2006.

Zhang, M., Uno, I., Yoshida, Y., Xu, Y., Wang, Z., Akimoto, H., Bates, T., Quinn, T., Bandy, A., and Blomquis, B.: Transport and transformation of sulphur compounds over East Asia during the TRACE-P and ACE-Asia campaigns, Atmos. Environ., 38, 6947-6959, 2004.

Zhang, W., Zhuang, G., Huang, K., Li, J., Zhang, R., Wang, Q., Sun, Y., Fu, J., Chen, Y., Xu, D., and Wang, W.: Mixing and transformation of Asian dust with pollution in the two dust storms over the northern China in 2006, Atmos. Environ., 44, 3394-3403, 2010 .
Zhang, X. Y., Wang, Y. Q. Wang, D. Gong, S. L. Arimoto, R., Mao, L. J., and Li, J.: Characterization and sources of regional-scale transported carbonaceous and dust aerosols from different pathways in coastal and sandy land areas of China, J. Geophys. Res.Atmos., 110, D15301, doi:10.1029/2004JD005457, 2005.

Zhao, Y., Wang, S., Duan, L., Cao, P., and Hao, J.: Primary air pollutant emissions of coal-firepower plants in China: current status and future prediction, Atmos. Environ., 42, 8442-8452, 2008.

Zheng, M., Wang, F., Hagler, G. S. W., Hou, X., Bergin, M., Cheng, Y., Salmon, L. G., Schauer, J., Louie, P., Zeng, L., and Zhang, Y.: Sources of excess urban carbonaceous aerosol in the Pearl River Delta Region, China, Atmos. Environ., 45, 1175-1182, 2011. 